\title{
DOE/PC/79963--T/5
}

DOE/PC/79903--T15

DE93 001869

\section{OPTICAL PROPERTIES OF FLYASH}

Contract No. DE-AC22-87PC 79903

Quarterly Report for Period 1 January - 31 March 1990

Prepared for Pittsburgh Energy Technology Center

Princlpal Investigator Professor S. A. Self

\section{DISCLAIMER}

This report was prepared as an account of work sponsored by an agency of the United States Government. Neither the United States Government nor any agency thereof, nor any of their
April $1990 \begin{aligned} & \text { employees, makes any warranty, express or implied, or assumes any legal liability or responsi- } \\ & \text { bility for the accuracy, completeness, }\end{aligned}$ bility for the accuracy, completeness, or usefulness of any information, apparatus, product, or process disclosed, or represents that its use would not infringe privately owned rights. Reference herein to any specific commercial product, process, or service by trade name, trademark, manufacturer, or otherwise does not necessarily constitute or imply its endorsement, recommendation, or favoring by the United States Government or any agency thereof. The views and opinions of authors expressed herein do not necessarily state or reflect those of the United States Government or any agency thereof.

\section{HIGH TEMPERATURE GASDYNAMICS LABORATORY Mechanical Engineering Department Stanford University




\section{OPTICAL PROPERTIES OF FLYASH \\ Contract No. DE-AC22-87PC 79903 \\ Quarterly Report for Period 1 January - 31 March 1990 \\ Prepared for Pittsburgh Energy Technology Center \\ Principsi Investigator Profossor S. A. Self}

\section{EXECUTIVE SUMMARY}

The general aims of this research are to provide a fundamental scientific basis for the physical understanding and reliable calculation of radiative heat transfer in coal combustion systems, particularly as it is influenced by the presence of inorganic constituents deriving from the mineral matter in coal.

The work is organized under four tasks. Tasks I and II were initiated in October 1987; Tasks III and IV were funded from October 1988.

Task 1. Characterization of Flyash: Under this heading the chemical composition and size distribution of representative flyashes are being measured by appropriate microanalytical techniques to provide information required in Task 2.

Task 2. Measurements of the Optical Constants of Slags: Under this heading measurernents of the infrared optical constants (i.e., the complex refractive index $m=n-i k$ ) of synthetic slags are being made as a function of wavelength and temperature for controlled compositions. Particular attention will be given to the contribution of the $\mathrm{Fe}_{2} \mathrm{O}_{3}$ content and its valence state. The data is being reduced to yield formulae giving the complex refractive index over relevant ranges of wavelength and temperature, as a function of the relevant metal oxide constituents.

Task 3. Sample Calculations of the Radiant Properties of Flyash Dispersions: This component comprises various calculations to guide and evaluate the experimental work under the other three tasks.

Task 4. Measurement of the Radiant Properties of Flyash Dispersions: This benchscale experiment is planned to compare the measured radiant properties of a dispersion of well-characterized ash with computations based on data developed under the first two tasks.

In this tenth quarter good progress has been made in all four areas, as reported in the Quarterly Report, and summarized below. 


\section{Task 1}

The ashes being characterized are samples from power plants or pilot-scale combustors derived from combustion of six of the coals selected for study under the parallel PETC program on "Transformation of Inorganic Coal Constituents in Combustion Systems."

The principal features requiring characterization are particle size and composition distributions, including correlations between size and composition. Size distributions are being measured in house by Coulter counter. Size and composition distributions are being determined by automated SEM/microprobe analysis at UNDERC. Other in-house characterization work under way includes size classification by wet sieving combined with classification by density using flotation/sedimentation techniques, low temperature ashing for the char content and magnetic separation for the magnetite content resulting from combustion of pyrite.

Size distributions for all six ashes over the range $0.5-60 \mu \mathrm{m}$ have been completed using the Coulter multisizer with a technique employing two orifices to cover the whole range. A suitable technique for matching distributions using two orifice sizes was devised. The measured distribution was found to be very well represented by truncated log-normal distributions. Comparison with the size distribution from automated SEM shows some discrepancy which is still being investigated.

Micrographs of the ash samples prepared by UNDERC for automated SEM/EDX sizecomposition analysis revealed many agglomerates, casting some doubt on the validity of the data. A freeze-drying technique for sample preparation has been developed at Stanford which gives very well dispersed samples. The automated SEM/EDX analysis has been repeated using such samples and the results have been analyzed using statistical computer codes developed at Stanford. Ternary diagrams of the composition distributions (in color) have been prepared using software developed at Stanford. In the past quarter, automated SEM/EDX analyses have been completed and preliminary analysis of the data made for all six ashes.

In addition, density classification results have been obtained for all six ashes and compared with results from SEM analysis for composition, which, of course, determines the density.

\section{Task 2}

Methods for determining the infrared optical properties of solid synthetic and natural slags at low temperatures have been established in prior work at Stanford. In the present work the main effort has been devoted to the development of suitable apparatus and 
techniques for performing similar measurements on slags at temperatures to $2000 \mathrm{~K}$, when the slag is liquid. Basic experimental strategies have been decided and apparatus has been designed to accomplish this task. Preliminary tests at high temperatures during past quarters have resulted in the first reliable measurements of the infrared absorption of liquid slag.

Two complementary techniques involving infrared optical measurements on liquid samples of synthetic slag maintained in an electric furnace have been developed. The first, for the wavelength range $1-5 \mu \mathrm{m}$ where the absorption index is low $\left(\mathrm{k} \leq 10^{-2}\right)$ employs a submerged platinum mirror to measure the absorption of thin films of slag by a double-pass technique. The second, applicable over the whole wavelength range $(1-12 \mu \mathrm{m})$, measures the surface reflectance of the liquid slag relative to that of a cold gold mirror in an external reference path.

The first technique has been successfully used to obtain the first reliable measurements of the infrared absorption of liquid slag (at $2000 \mathrm{~K}$ ).

Efforts have since been concentrated on developing and testing the second technique (for surface reflectivity measurements). It was determined that the quality of the data obtained is limited by the differing absorptions due to $\mathrm{CO}_{2}$ and $\mathrm{H}_{2} \mathrm{O}$ in the hot measurement path and the cold reference path. To eliminate this problem, the whole apparatus has been enclosed in a chamber, purged with dry nitrogen.

With this modification, good measurements of the reflectivity of synthetic slag containing $5 \% \mathrm{Fe}$ at $1600^{\circ} \mathrm{C}$ were made over the whole wavelength range $1-12 \mu \mathrm{m}$. The data in the range 8-12 $\mu \mathrm{m}$ were reduced, using the Kramers-Kronig technique to give both the real and imaginary parts of the complex refractive index.

Possible sources of uncertainty in these measurements have been critically investigated and resolved. In particular, a problem due to contamination of a mirror by furnace gases has been identified and steps taken to eliminate it by redesign of the optical system. Also, in this quarter, reflectance measurements on liquid slags containing zero, $1 \%$ and $5 \%$ iron (as $\mathrm{Fe}_{2} \mathrm{O}_{3}$ ) have been completed. The results have been reduced, using $\mathrm{Kramers}-\mathrm{Kronig}$ analysis to yield the real $(n)$ and imaginary $(k)$ parts of the complex refractive index.

\section{Task 3}

Programs have been written for Mie scattering calculations which are then convolved with input on the size and optical constants distributions for a particulate dispersion to yield the spectral scattering and absorption coefficients of the aerosol. Additionally, a prograris has been written to solve the radiation transfer problem for a homogeneous slab, utilizing the 
exact solution method of Case's normal modes. Input for the spectral scattering and absorption coefficients from the first program allows the spectral scattering, absorption and emission properties of the slab to be computed. These can then be integrated over wavelength to yield the total radiative heat transfer characteristics of the slab.

These programs have been used to determine the importance of certain features of typical ashes for radiation transfer. These include the sensitivity of the optical/radiative properties of a flyash dispersion to (i) composition-size correlation, especially with regard to the distribution of iron oxides with particle size, and (ii) the presence of bubbles in the glassy ash particles.

This computational capability is also being used to evaluate the experimental conditions in the design of the apparatus for Task 4.

\section{Task 4}

Careful consideration has been given to the feasibility of various basic approaches for implementing the goals of this task. After evaluating various experimental techniques, a basic approach has been identified, which involves extinction measurements on flyash dispersed in suitable organic liquids. Measurements of the infrared transmission of three selected liquids have been made which confirm their suitability for this purpose. CaF2 windows for an absorption cell have been acquired and preliminary tests of a suitable cell design have been made. 


\subsection{INTRODUCTION}

This is the tenth quarterly report under DOE contract No. DE-AC22-87PC 79903 entitled "Optical Properties of Flyash." Tasks 1 and 2 of this program were funded from 15 September 1987. Tasks 3 and 4 were funded from 15 September 1988.

The general aims of this research are to provide a fundamental scientific basis for the physical understanding and reliable calculation of radiative heat transfer in coal combustion systems, particularly as it is influenced by the presence of inorganic constituents deriving from the mineral matter in coal. Some preliminary work in this area has been carried out at Stanford in the past several years with NSF support. The present program will greatly enlarge the scope of this work.

The complete, integrated program of theoretical and experimental work comprises four separate tasks.

Task 1. Characterization of Flyash

Task 2. Measurements of the Optical Constants of Slags

Task 3. Sample Calculations of the Radiant Properties of Flyash Dispersions.

Task 4. Measurements of the Radiative Properties of Flyash Dispersions.

In Task 1, the chemical composition and size distribution of representative flyashes are being measured by appropriate microanalytical techniques to provide information required in Tasks 2 and 3.

In Task 2, measurements of the infrared optical constants (i.e., the complex refractive index $m=n-i k$ ) of synthetic slags are being made as a function of wavelength and temperature for controlled compositions. Particular attention is being given to the contribution of $\mathrm{Fe}_{2} \mathrm{O}_{3}$ content and its valence state. The data will be reduced to yield formulae giving the complex refractive index over relevant ranges of wavelength and temperature, as a function of the relevant metal oxide constituents.

In Task 3, sample calculations are being made for typical ash loadings, size distributions and compositions for simple geometries, with two main purposes: first, to provide insight and physical understanding of the role of flyash in radiative heat transfer in combustion systems; second, to indicate the sensitivity of the results to the characteristics of the input data. Such calculations will also be used to determine appropriate conditions and to predict the expected measured radiative properties for the experiment of Tasi 4. 
The experiment of Task 4 is designed to critically test our ability to predict the measured spectral emittance and scattering coefficient of flyash dispersions under wellcontrolled laboratory conditions utilizing the optical property data developed in Task 2. Particular attention will be paid to assessing the contribution of the char component in typical ashes. Any discrepancies between calculated and measured quantities revealed by these tests will be resolved by appropriate further studies.

A more detailed description of the scope of these tasks is given below. First, rowever, an outline is given of the rationale for the overall approach adopted in this program.

\subsection{Rationale of Overall Approach}

To account for the effects of flyash in radiative heat transfer calculations requires a knowledge of the contributions of the ash to the spectral absorption $\left(a_{\lambda}\right)$ and scattering $\left(\sigma_{\lambda}\right)$ coefficients of the particulate dispersion, together with the phase function $\Phi_{\lambda}$ describing the anisotropy of the scattering. These quantities depend on the particulate loading as well as the distributions of the size and optical properties of the particles.

For a spherical particle of homogeneous, optically isotropic material, characterized by a complex refractive index $m \equiv(n-i k)$ Mie theory allows one to compute the spectral absorption $\left(Q_{\lambda, a}\right)$ and scattering $\left(Q_{\lambda, s}\right)$ efficiencies of the particle, as well as the phase function $\phi_{\lambda}$. For randomly polarized radiation, these quantities are a function of the particle size parameter $x \equiv(\pi d / \lambda)$, and the complex refractive index $m(C, \lambda, T)$, a function of composition, wavelength and temperature.

For a monodispersion of identical spherical particles, of specified loading (i.e. number density), the particulate's contribution to the optical properties $\left(a_{\lambda}, \sigma_{\lambda}\right.$ and $\left.\Phi_{\lambda}\right)$ of the medium are simply related to the spectral properties $\left(Q_{\lambda_{1}}, Q_{\lambda_{1}}, \phi_{\lambda}\right)$ of a single particle. It is also straightforward to compute the spectral optical properties of the medium for a polydispersion of spheres of identical composition, by convolving the results of Mie calculations for spheres of varying diameter (i.e. $x$ ) for fixed wavelength (and hence fixed $m$ ), with the particle size distribution (assumed given). In the case of a particulate material, like flyash, for which it is reasonable to assum that individual particles are of homogeneous composition but the composition varies frum particle to particle, it is still possible to compute the spectral characteristics of the particulate dispersion by dividing the particles into an appropriate number of classes of varying composition (and hence $m$ ), each having a specified size distribution, and summing over particle classes. 
In radiative heat transfer calculations, the contribution of the gas to the spectral absorption coefficient is added to that of the particles to obtain the combined optical properties of the medium on a spectral basis. These optical properties are then used as inputfor a radiation transfer code to calculate radiative fluxes, on a spectral basis, for a particular combustor geometry and boundary conditions. Finally, to obtain total heat transfer quantities such as the overall radiant heat flux, integrations over wavelength must be made.

The procedure, outlined above, represents the only logical approach to the computation of radiative heat transfer in flyash laden combustion gases. To implement this procedure requires, as input, a detailed characterization of the ash with respect to its size and (complex) refractive index distributions on a spectral basis.

Now, while techniques are available for determining the size distribution of powder samples, such as flyash, there are no practical means available for reliably determining the complex refractive index distribution of a complex material such as flyash either on a single particle basis, as a powder or as a dispersed aerosol. However, it is possible, using modern microanalytical techniques, specifically computer-automated SEM/EDX analysis, to determine the size and chemical composition of a heterogeneous powder on a particle by particle basis for a statistically large number of particles.

If the compositions of individual particles can be related to the complex refractive index of their material, then the characterization of a particular ash in terms of its size and composition distributions can lead to the necessary input for carrying out the calculations, outlined above, to compute radiation transfer in combustion systems containing that ash.

Thus the key requirement, necessary for the implementation of this approach, is data on the optical constants (i.e. the components $n, k$ of the complex refractive index) as a function of composition, wavelength and temperature covering the range of compositions found in representative ashes. Since, as noted above, and emphasized in texts on the optical properties of particulate matter, it is impractical to extract reliable data on the optical constants of material in particulate form, the only viable approach is to make measurements on homogeneous bulk samples for which well-established techniques are available.

The foregoing arguments provide the rationale for the present program. Characterization of representative flyashes concerning their size and composition distributions constitutes Task 1 , while measurements of the optical constants on bulk samples of synthetic slags as a function of relevant ranges of composition, wavelength and temperature constitute Task 2. Task 3 is designed to provide computational capabilities to support the other tasks, while Task 4 is planned to provide an experimental test that the measured optical properties of a dispersion of flyash can indeed be computed reliably from a knowledge of the size and composition distributions of the ash. 


\subsection{Description of Tasks}

\section{TASK 1 - Characierization of Elyash}

Extensive prior analyses of flyash from a wide range of coals plus analyses of the mineral matter in raw coals, together with knowledge of the transformation processes occurring during combustion, lead to the following overall picture of the nature of flyash.

The particle size distribution is very broad with a volume (or mass) mean diameter on the order of $10 \mu \mathrm{m}$. Typically it is well represented by a log normal distribution with the $1 \%$ and $99 \%$ sizes in a cumulative plot by volume occurring at $\sim 1 \mu \mathrm{m}$ and $70 \mu \mathrm{m}$ respectively. Evidence of a distinct submicron fume due to homogeneous condensation of volatile mineral matter is sometimes found, but this fraction can be expected to contribute negligibly to radiation transfer.

With regard to chemical composition, several distinct classes of particle can be identified and plausibly related to their origin and formation mechanisms.

By far the preponderant class, usually representing on the order of $90 \%$ or more of the ash on a mass basis, consists of vitreous (amorphous) material composed primarily of $\mathrm{SiO}_{2}$, $\mathrm{Al}_{2} \mathrm{O}_{3}, \mathrm{CaO}$ and $\mathrm{MgO}$, usually in that order, but containing varying smaller percentages of other metal oxides, notably $\mathrm{Fe}_{2} \mathrm{O}_{3}$. It can appropriately be identified as particles of impure (calcium) aluminosilicate glass derived from the microscopic clay-like mineral inclusions in the coal matrix. As char burnout proceeds these inclusions melt and form liquid globules on the surface of the char (which they do not wet) and are then released into the gas.

These glassy particles tend to be quite spherical with smooth surfaces and of reasonably homogeneous composition as is to be expected from their formation as liquid droplets. As they cool after release from the char surface, they remain in the vitreous state because the cooling rate is much faster than the crystallization rate for the formation of specific phases. The fact that the bulk of most ashes consists of reasonably spherical, homogeneous and vitreous (and therefore optically isotropic) particles is a very fortunate fact, since they satisfy the assumptions of the Mie theory remarkably well.

Micrographs of optically polished sections of ash cast in epoxy resin, shows that these glassy particles sometimes contain a number of small bubbles of gas evolved from the char and trapped in the particles as they form on the char surface. More rarely, large, thin-walled cenospheres are observed which presumably are "glass-blown" when a liquid drop covers a pore in the char from which a relatively large volume of gas is evolved under pressure. Although such cenospheres are very prominent objects in micrographs, their number is usually too small to significantly affect radiation transfer. 
Auger spectroscopic studies of ash often show a thin surface layer composed of volatile metals and high in sulfur (as sulfates) and water. The presence of a thin layer of adsorbed water containing sulfate ions controls the electrical resistance of the surface which is very important in the performance of electrostatic precipitators. However, this surface layer, of different composition from the underlying particle, is too thin $(\leq 100 \AA)$ relative to wavelengths of interest to affect the optical properties of the particle.

Apart from this major class of glassy particles, several distinct minor classes of particle types can be identified, each comprising, at most, a few percent by mass of the flyash. One such class consists of incompletely burned char particles which are clearly identified in optical and SEM micrographs by the fact that they are black, of irregular shape and porous. The mass fraction of char depends on the particular coal and the combustor configuration and operating conditions. In modern combustors the mass fraction of unburned char is normally a few percent at most.

Another minor class consists of particles of adventitious incombustible mineral matter (e.g. quartz) which is contained in the pulverized coal feed. Such particles are usually large and of irregular shape, often showing rounded edges indicating partial melting.

A third minor class consists of magnetite $\left(\mathrm{Fe}_{3} \mathrm{O}_{4}\right)$ which derives from the combustion of pyrite $\left(\mathrm{FeS}_{2}\right)$ particles contained in the coal grind. These magnetite particles are black, generally spherical, magnetic and much denser than the glassy particles. The proportion of magnetite particles depends on the coal type, being largest in high sulfur coals, because the sulfur is mostly associated with pyrite. Recent work has shown that much of the pyrite in the coal grind can be removed by washing/sedimentation with a reduction in $\mathrm{SO}_{\mathbf{x}}$ emissions as high as $50 \%$ in some high sulfur coals.

The ashes selected for characterization are samples from power plants or pilot-scale combustors derived from the same seven coals selected for study under the parallel PETC program on "Transformation of Inorganic Coal Constituents in Combustion Systems" which comprise four bituminous, one sub-bituminous and two lignite coals.

A variety of techniques are being used in the characterization of these ashes. The principal method for determining size distributions employs a Coulter Multisizer which is capable of giving accurate, reliable results of high resolution over a wide dynamic range $\leq 1 \mu \mathrm{m}$ to $\geq 100 \mu \mathrm{m}$.

Automated, computer-controlled, combined SEM/EDX microanalysis will be the principal technique used to determine the distributions of composition and size for a large number $(-1000)$ of particles for each ash. The size distributions will be compared with those obtained by the Coulter counter. 
Other techniques to be used include classification by density using liquids of varying density in a centrifuge, together with classification by size using a wet-sieving method. These techniques can yield density and size separated fractions for further examination by microanalytical techniques such as energy-dispersive $X$-ray spectroscopy. In addition, the magnetite particles may be separated by magnetic separation. The char content will be determined by low temperature ashing.

\section{TASK 2 - Measurements of Optical Constants of Synthetic Slags}

This task is planned to provide the basic optical properties data in a comprehensive and conveniently usable form. The optical constants (i.e., the components of the complex refractive index $m=n-i k$ ) of samples of synthetic slags of controlled compositions will be measured using established techniques involving transmission and surface reflectance methods. The wavelength range will extend from the visible to $12 \mu \mathrm{m}$, and the temperature range will extend to $2000 \mathrm{~K}$.

In earlier work at Stanford, supported by NSF, extensive measurements of this type were made on polished wafers of synthetic slags at temperatures up to $1200 \mathrm{~K}$. A major component of this task will be to extend such measurements to higher temperatures $(\sim 2000 \mathrm{~K})$ where the slag is liquid. This requires the development of modified techniques which present a number of more or less severe technical challenges.

Initially, the optical constants of the basic calcium-aluminosilicate host glass will be determined for the composition range defined by Task 1. Subsequently, by adding infraredactive mineral oxide constituents in controlled amounts, one at a time, the modifications to $\mathrm{m}(\lambda, \mathrm{T})$ produced by such constituents will be quantitatively determined. The particular constituents (and their range of mass fractions) to be examined will be determined by those disclosed by Task 1, taking account of knowledge of the optical activity at relevant wavelengths of such additions from the literature of glass technology. Specific constituents to be examined will include $\mathrm{Fe}_{2} \mathrm{O}_{3}$, taking especial account of its valence state $\left(\mathrm{Fe}^{2+} / \mathrm{Fe}^{3+}\right.$ ratio), and of $\mathrm{TiO}_{2}$. The contribution of the $\mathrm{OH}$ radical to the optical properties will be evaluated and quantified if significant.

The experimental data on $\mathrm{m}(\lambda, \mathrm{T})$ as a function of composition, over the range relevant to coal ashes, will be reduced to generate simple correlation formulae. The latter will constitute the data base necessary to calculate the radiative properties of bulk slags and ash dispersions required for understanding and computing radiative transfer in coal combustion systems. 


\section{TASK 3 - Sample Calculations of the Radiant Properties of Flyash Dispersions}

This task is intended to provide computational capabilities to support the other tasks. It includes the following components.

(i) A Mie scattering code to calculate the absorption and scattering efficiencies and phase function of a single sphere of specified size parameter and complex refractive index. A modified Mie code will also allow such computations for hollow spheres.

(ii) A code to convolve the results from (i) over a specified size distribution and loading to compute the absorption and scattering coefficients and phase function of a homogeneous polydispersion.

(iii) A code to sum the results of (ii) for a number of classes of particles of varying refractive indices and size distributions, i.e. for a heterogeneous polydispersion.

(iv) A radiation transfer code to calculate the absorption, scattering and emission characteristics of a homogenous, isothernal slab of dispersed ash on a spectral basis.

(v) A code to integrate the spectral results from (iv) over wavelength to yield the total radiative properties of the slab.

Calculations using these codes will be used to provide sensitivity analyses to guide the characterization work of Task 1, and to design and evaluate the results of Task 4.

\section{TASK 4 - Measurement of the Radiative Properties of Flyash Dispersions}

The purpose of this laboratory scale experiment is to test our ability to predict the measured radiative properties of a dispersion of well-characterized flyash. As such it will provide a critical test of the effectiveness of the overall approach adopted in this program. 


\subsection{PROGRESS IN THE PAST QUARTER}

\subsection{Task 1: Characterization of Fly Ash}

\section{Preparation of Slag Samples and Measurement of Slag Densities:}

Samples of each fly ash, weighing 40 to $50 \mathrm{gm}$, (all from baghouses, except one sample from the cyclone for the Beulah ash) were melted to make slags. The melts were prepared in alumina crucibles, in the oven used in Task 2. The slag was kept molten for more than fourteen hours before a small amount was withdrawn from the crucible using a long alumina rod. The rapid quenching resulted in the formation of homogeneous, glassy slag, some of which will be used as samples for microprobe study. The slag was cooled in the oven; the temperature decreasing linearly at about $10^{\circ} \mathrm{C}$ per minute. In many cases, this cooling rate was not sufficiently high to prevent some crystallization. However, the slag is sufficiently homogeneous to allow accurate density measurement to be made as described later in this section. The major source of error in composition measurement of slag prepared in this way is a slight over-estimation of the alumina content arising from the dissolution of small amounts of the alumina crucible in the melt.

Satisfactory slags have not yet been obtained from two ashes. The Eagle Butte ash, with its high calcia content, fluxes at lower temperatures - and, in the process, becomes contaminated by dissolving a large portion of the alumina crucible in contact with the liquid slag. A chemically purer sample will be prepared next quarter in a platinum crucible. The Illinois \#6 slag was found to contain a large pellet of iron at the bottom of the crucible. It is thought that the oxidation of the small amount of char in the ash produced carbon-monoxide, which, in turn, reduced the magnetite to iron. The heavier iron sank to the bottom of the crucible, and, due to surface tension effects, formed a globular mass. Consequently, the bulk slag will show a lower iron content than the actual ash. We are currently working on a better way to prepare the slag sample for this ash.

The densities of the slags were measured by a standard procedure using the mass of the slag, the mass of an accurately measured volume of fluid (water in this case) and the mass of the same volume of fluid and the slag together. A specific gravity bottle was used for the volume measurement. The following formula is used:

$$
\text { slag density }=\frac{c(a-d)}{v(a+c-b)}
$$


where $\quad a=$ weight of the specific gravity bottle (capacity $\mathrm{V} \mathrm{ml}$ ) filled with the fluid;

b = weight of the same bottle filled with the fluid plus the slag;

$c$ = weight of the dry slag;

d = weight of the dry, empty, specific gravity bottle.

The results of the density measurements are presented in the next section. Three sets of nizasurements were made for each slag. The variation of the values of the densities obtained were within $\pm 3 \%$. The measurement technique being very accurate, this variation is probably due to non-uniformity in the composition of the slag or due the presence of small cavities in the slag created by trapped gases.

\section{Correlation of Density Classification Data of Ash with Bulk Slag Density Data:}

A bulk density for each fly ash was computed from the centrif ly? separation data in the following manner. Among the major constituents of any ash part ile, the one with the lowest density is silica, with a density of $2.2 \mathrm{~g} / \mathrm{cc}$ (see QPR for period ending January, 1989 for the densities of the oxides of the major elements of fly ash). Very few of the ash particles are purely silica, and the density of the next major constituent, alumina, is much higher $(4.0 \mathrm{~g} / \mathrm{cc})$. Hence, it is reasonable to assume that all ash in the first three density classes $(<1.6 \mathrm{~g} / \mathrm{cc}-2.4 \mathrm{~g} / \mathrm{cc})$ are, to varying degrees, cenospheric. Inclusion of these classes in the calculation of an average density would lead to misleading results. For this reason, the bulk density was calculated using the data for the last three density classes $(2.4 \mathrm{~g} / \mathrm{cc}->3.2 \mathrm{~g} / \mathrm{cc})$. The fraction of the ashes in these three classes constitute between $49 \%$ to $96 \%$, by mass, of the whole ashes - with the prominent exception of the Texas lignite, where it accounts for only $4 \%$. By omitting the mass fraction of ash in cenospheres, we are effectively assuming that the this fraction has the same average composition as that of the rest of the ash. This average density is calculated using the formula:

$$
\rho_{\mathrm{av}}=\frac{\mathrm{mf} 4+\mathrm{mf5}+\mathrm{mf6}}{\frac{\mathrm{mf} 4}{2.6}+\frac{\mathrm{mf5}}{3.0}+\frac{\mathrm{mf6}}{5.2}}
$$

where $\mathrm{mf} 4, \mathrm{mf5}$ and $\mathrm{mf} 6$ are the mass fractions in the highest three density classes. It has been found that the density class $>3.2 \mathrm{~g} / \mathrm{cc}$ is almost entirely made up of magnetite, of density $5.2 \mathrm{~g} / \mathrm{cc}$. For the density classes $2.4-2.8 \mathrm{~g} / \mathrm{cc}$ and $2.8-3.2 \mathrm{~g} / \mathrm{cc}$, the mid-points $2.6 \mathrm{~g} / \mathrm{cc}$ and $3.0 \mathrm{~g} / \mathrm{cc}$ were used. The results are tabulated below. Bearing in mind the extent of the 
assumptions involved, it is interesting to note that the average density is quite close to the slag densities measured as described in the previous section.

\section{Table 1}

\begin{tabular}{|c|c|c|c|c|}
\hline Source of flyash & $\begin{array}{l}\text { Percentage of flyash, } \\
\text { by mass, that are } \\
\text { cenospheric (hollow) }\end{array}$ & $\begin{array}{c}P_{\mathrm{av}} \\
(\mathrm{g} / \mathrm{cc})\end{array}$ & $\begin{array}{c}\rho_{s} \\
(g / c c)\end{array}$ & $1-\frac{\rho_{\mathrm{av}}}{\rho_{\mathrm{s}}}$ \\
\hline Kentucky \#9 & 35.25 & 2.82 & 2.80 & $-0.71 \%$ \\
\hline Illinois \#6 & 51.22 & 2.73 & 2.69 & $-1.49 \%$ \\
\hline Eagle Butte & 4.19 & 2.98 & 2.89 & $-3.11 \%$ \\
\hline Upper Freeport & 34.94 & 2.73 & 2.67 & $-2.25 \%$ \\
\hline Beulah (Baghouse) & 12.54 & 2.67 & 2.98 & $10.40 \%$ \\
\hline Beulah (Cyclone) & 36.35 & 2.90 & 2.92 & $0.68 \%$ \\
\hline
\end{tabular}

The San Miguel ash is treated somewhat differently. The average density is computed from the centrifugal separation data using all six density categories (average density of the lightest class, $<1.6 \mathrm{~g} / \mathrm{cc}$, is assumed to be $1.4 \mathrm{~g} / \mathrm{cc}$ ) and found to be $1.68 \mathrm{~g}$ 'cc. This is much lower than the slag density of $2.39 \mathrm{~g} / \mathrm{cc}$. By examining polished sections of San Miguel ash particles embedded in epoxy, we have observed that almost all the ash particles are hollow some with multiple and interconnected cavities. Considering that Mie computations for such complex geometries are not practically feasible, the following simplified model is proposed for this ash:

(1) all the particles are cenospheric;

(2) the ratio, $R$, of the inner diameter to the outer diameter of the cenosphere is the same for all particles. This ratio is computed using the formula:

$$
R \equiv \frac{D_{\text {inner }}}{D_{\text {outer }}}=\left(1-\frac{\rho_{a v}}{\rho_{s}}\right)^{1 / 3}
$$

where $\rho_{\mathrm{s}}$ and $\rho_{\mathrm{av}}$ are the slag and average ash densities, respectively. The formula can easily be derived by equating the masses computed for a particle using the two densities. Since for the San Miguel ash $\rho_{a v}=1.68 \mathrm{~g} / \mathrm{cc}$ and $\rho_{\mathrm{s}}=2.39 \mathrm{~g} / \mathrm{cc}$, we find $R=0.67$. 
assumptions involved, it is interesting to note that the average density is quite close to the slag densities measured as described in the previous section.

Table 1

\begin{tabular}{|c|c|c|c|c|}
\hline Source of flyash & $\begin{array}{l}\text { Percentage of flyash, } \\
\text { by mass, that are } \\
\text { cenospheric (hollow) }\end{array}$ & $\begin{array}{c}\rho_{\mathrm{av}} \\
(g / c c)\end{array}$ & $\begin{array}{c}p_{3} \\
(g / c c)\end{array}$ & $1-\frac{\rho_{\mathrm{av}}}{\rho_{\mathrm{s}}}$ \\
\hline Kentucky \#9 & 35.25 & 2.82 & 2.80 & $-0.71 \%$ \\
\hline Illinois \#6 & 51.22 & 2.73 & 2.69 & $-1.49 \%$ \\
\hline Eagle Butte & 4.19 & 2.98 & 2.89 & $-3.11 \%$ \\
\hline Upper Freeport & 34.94 & 2.73 & 2.67 & $-2.25 \%$ \\
\hline Beulah (Baghouse) & 12.54 & 2.67 & 2.98 & $10.40 \%$ \\
\hline Beulah (Cyclone) & 36.35 & 2.90 & 2.92 & $0.68 \%$ \\
\hline
\end{tabular}

The San Migue! ash is treated somewhat differently. The average density is computed from the centrifugal separation data using all six density categories (average density of the lightest class, $<1.6 \mathrm{~g} / \mathrm{cc}$, is assumed to be $1.4 \mathrm{~g} / \mathrm{cc}$ ) and found to be $1.68 \mathrm{~g} / \mathrm{cc}$. This is much lower than the slag density of $2.39 \mathrm{~g} / \mathrm{cc}$. By examining polished sections of San Miguel ash particles embedded in epoxy, we have observed that almost all the ash particles are hollow some with multiple and interconnected cavities. Considering that Mie computations for such complex geometries are not practically feasible, the following simplified model is proposed for this ash:

(1) all the particles are cenospheric;

(2) the ratio, $R$, of the outer diameter to the inner diameter of the cenosphere is the same for all particles. This ratio is computed using the formula:

$$
T=3 \sqrt{1-\frac{P_{\mathrm{av}}}{\rho_{\mathrm{s}}}}
$$

where $\rho_{\mathrm{s}}$ and $\rho_{\mathrm{av}}$ are the slag and average ash densities, respectively. The formula can easily be derived by equating the masses computed for a particle using the two densities. Since for the San Miguel ash $\rho_{a v}=1.68 \mathrm{~g} / \mathrm{cc}$ and $\rho_{\mathrm{S}}=2.39 \mathrm{~g} / \mathrm{cc}$, we find $R=0.67$. 


\subsubsection{Microanalysis by SEM/EDX:}

As reported in the last QPR, concentrations of $\mathrm{Na}$ and $\mathrm{Cl}$ much larger than expected were found in the composition data of the four fly ashes (San Miguel, Eagle Butte, Beulah, Upper Freeport) sent to UNDERC (University of Norti Dakota Energy Research Center) for CCSEM (computer controlled scanning electron microanalysis). The ratio of $\mathrm{Na}$ to $\mathrm{Cl}$, in particles with concentrations greater than $5 \%$ fo. either elements, is close to that in $\mathrm{NaCl}$. It was concluded that the samples got contaminated during preparation by $\mathrm{NaCl}$ solution which later crystallized on the ash particles. New samples of the four ashes have been prepared and sent to UNDERC for fresh analyses. However, we have re-analyzed the existing data after rejecting those particles having concentrations of $\mathrm{Na}$ and $\mathrm{Cl}$ greater than 5\% (Figure 1). Not surprisingly, the concentrations of the other elements have increased when compared to the anciyses presented in the last QPR. The trenois for elemental distribution as a function of particle diameter has not changed for the Eagle Butte and San Miguel ashes. However, for the Upper Freeport ash, there is now much less variation in chemical composition as a function of size. That variation was an artifact introduced by varying amounts of $\mathrm{Na}$ and $\mathrm{Cl}$ impurities on different particles. Although the distribution for the new Upper Freeport data is similar to the old data (Figure !), there is considerably less iron and more aluminium in the newer data. One should recall that the older sample consisted primarily of aggregates of ash particles. For this reason, it is preferable to await the results of the fresh analysis before any serious comparison of data from two CCSEM analyses of the same ash is made. It is noted, however, that a considerable proportion of the iron in the ash can be present as pure magnetite particles that are few in number. In that case, a sample size of a thousand particles may not be large enough to accurately account for the iron.

The information on chemical composition as a function of particle diameter will be obtained over increments of one micron by averaging the compositions of all particles in a given range. The composition distribution for the Illinois \#6 ash is shown in Table 2. The number of particles in each size bin are shown next to the particle diameter. The accuracy of this composition distribution is limited by the small sizes of the samples, especially for diameters $>6 \mu \mathrm{m}$. These composition distributions will be converted to complex refractive index distributions using optical property data from Task 2. 

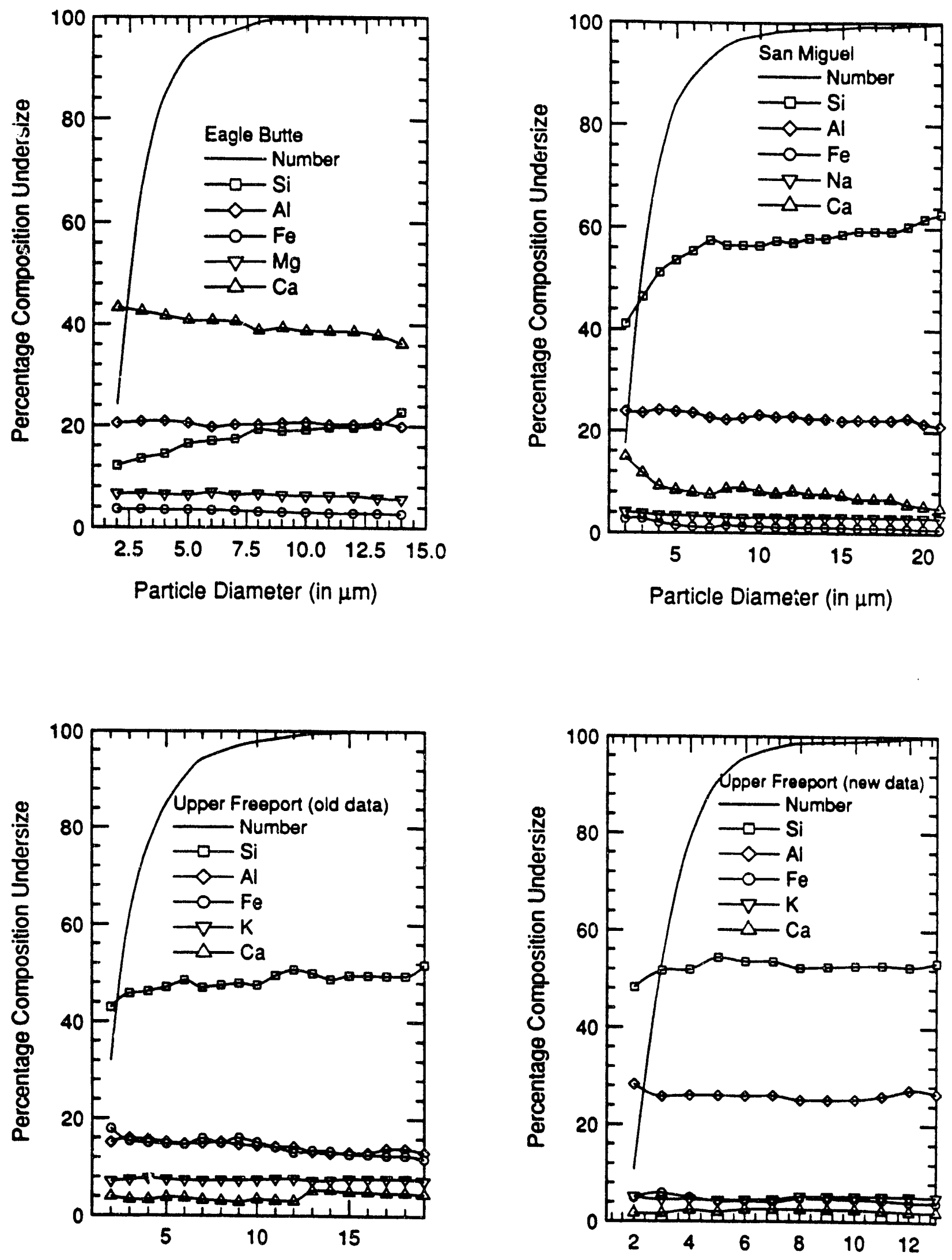

Particle Diameter (in $\mu \mathrm{m}$ )

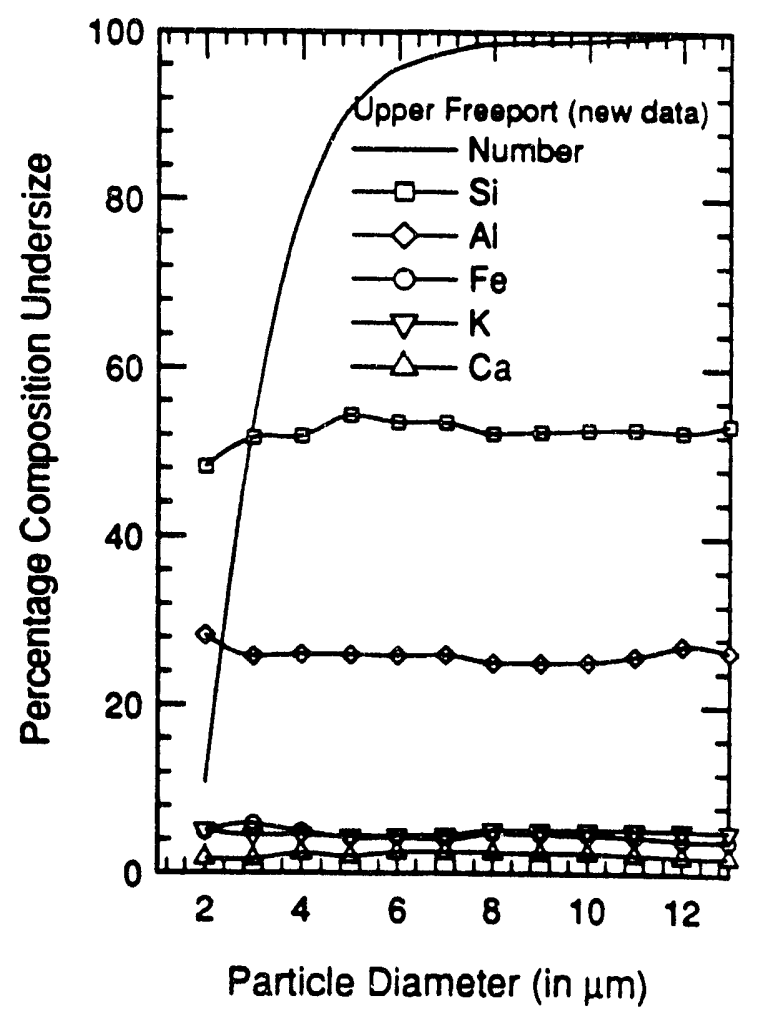

Figure 1: Average elemental composition of fly ash particles obtained from CCSEM 


\section{Table 2}

$\begin{array}{llllllllllllll}\text { Diameter Particles } & \mathrm{Si} & \mathrm{Al} & \mathrm{Fe} & \mathrm{Ca} & \mathrm{Na} & \mathrm{K} & \mathrm{Ba} & \mathrm{Ti} & \mathbf{M g} & \mathbf{P} & \mathrm{S} & \mathrm{Cl}\end{array}$

$\begin{array}{llllllllllllll}1 & 270 & 0.391 & 0.120 & 0.189 & 0.025 & 0.002 & 0.028 & 0.008 & 0.014 & 0.002 & 0.006 & 0.208 & 0.005 \\ 2 & 213 & 0.468 & 0.133 & 0.129 & 0.023 & 0.003 & 0.029 & 0.009 & 0.013 & 0.002 & 0.005 & 0.180 & 0.006 \\ 3 & 142 & 0.529 & 0.144 & 0.108 & 0.038 & 0.001 & 0.029 & 0.009 & 0.015 & 0.002 & 0.004 & 0.115 & 0.004 \\ 4 & 116 & 0.537 & 0.159 & 0.101 & 0.031 & 0.002 & 0.027 & 0.007 & 0.012 & 0.002 & 0.003 & 0.114 & 0.005 \\ 5 & 72 & 0.582 & 0.147 & 0.116 & 0.017 & 0.002 & 0.025 & 0.008 & 0.011 & 0.001 & 0.001 & 0.087 & 0.003 \\ 6 & 60 & 0.607 & 0.145 & 0.094 & 0.023 & 0.001 & 0.029 & 0.005 & 0.011 & 0.001 & 0.001 & 0.080 & 0.003 \\ 7 & 37 & 0.627 & 0.121 & 0.087 & 0.038 & 0.002 & 0.024 & 0.006 & 0.009 & 0.001 & 0.002 & 0.081 & 0.003 \\ 8 & 35 & 0.619 & 0.151 & 0.113 & 0.027 & 0.002 & 0.030 & 0.005 & 0.009 & 0.001 & 0.002 & 0.036 & 0.005 \\ 9 & 23 & 0.577 & 0.174 & 0.073 & 0.049 & 0.002 & 0.030 & 0.006 & 0.018 & 0.002 & 0.002 & 0.066 & 0.002 \\ 10 & 20 & 0.625 & 0.134 & 0.119 & 0.019 & 0.002 & 0.032 & 0.005 & 0.013 & 0.000 & 0.002 & 0.044 & 0.006 \\ 11 & 17 & 0.578 & 0.191 & 0.105 & 0.018 & 0.003 & 0.031 & 0.008 & 0.008 & 0.003 & 0.001 & 0.054 & 0.002 \\ 12 & 6 & 0.479 & 0.200 & 0.098 & 0.017 & 0.001 & 0.019 & 0.008 & 0.021 & 0.002 & 0.000 & 0.150 & 0.006 \\ 13 & 6 & 0.650 & 0.133 & 0.058 & 0.006 & 0.002 & 0.024 & 0.006 & 0.007 & 0.000 & 0.010 & 0.096 & 0.008 \\ 14 & 2 & 0.811 & 0.084 & 0.019 & 0.007 & 0.004 & 0.039 & 0.006 & 0.023 & 0.000 & 0.000 & 0.0 n & 0.008 \\ 15 & 1 & 0.693 & 0.201 & 0.023 & 0.000 & 0.000 & 0.026 & 0.018 & 0.027 & 0.000 & 0.000 & 0.013 & 0.000 \\ 16 & 0 & 0.000 & 0.000 & 0.000 & 0.000 & 0.000 & 0.000 & 0.000 & 0.000 & 0.000 & 0.000 & 0.000 & 0.000 \\ 17 & 4 & 0.620 & 0.219 & 0.025 & 0.023 & 0.000 & 0.032 & 0.012 & 0.034 & 0.000 & 0.000 & 0.027 & 0.008 \\ 18 & 3 & 0.656 & 0.218 & 0.031 & 0.008 & 0.002 & 0.030 & 0.010 & 0.006 & 0.000 & 0.000 & 0.027 & 0.013 \\ 19 & 1 & 0.548 & 0.077 & 0.183 & 0.008 & 0.000 & 0.021 & 0.039 & 0.039 & 0.010 & 0.000 & 0.058 & 0.017 \\ 20 & 0 & 0.000 & 0.000 & 0.000 & 0.000 & 0.000 & 0.000 & 0.000 & 0.000 & 0.000 & 0.000 & 0.000 & 0.000 \\ 21 & 1 & 0.960 & 0.000 & 0000 & 0.000 & 0.000 & 0.000 & 0.000 & 0.016 & 0.000 & 0.000 & 0.024 & 0.000\end{array}$




\subsection{Task 2: The Optical Properties of High Temperature Slag}

The immediate goal of this task is to measure the high temperature optical properties of coal slag. The complex refractive index, $m=n+i k$, is being measured for synthetic slags at high temperatures, near $1900 \mathrm{~K}$. The effect of composition on the optical properties is also being investigated.

In the previous QPR, measurements of the near normal reflectance of three different slag compositions were reported. From those measurements the complex refractive index, $m$, was computed using the Kramers-Kronig relations, with $n$ being determined for the wavelength range $1<\lambda<13 \mu m$ and $k$ being determined for a narrower range $(8<\lambda<13 \mu m)$.

During the past quarter, several new synthetic slags were made by melting mixtures of powdered oxides in an electric furnace at $1550^{\circ} \mathrm{C}$. During that process, the furnace was damaged when one of the molten slags dissolved its alumina container and flowed onto the alumina furnace tube. The furnace has since been repaired and is now operable again.

Reflectance and transmittance measurements are scheduled during the current quarter for the remaining synthetic slags planned for further study under this contract.

\subsection{Task 3: Sample Calculations of the Radiant Properties of Fly Ash Dis- persions}

During the past quarter, several new computer codes were developed to allow a broader investigation of the effects of fly ash on radiation heat transfer in coal combustion systems than was possible using previously developed codes [1].A more flexible radiation transfer calculation technique is used to allow a study of the effects of anisotropic scattering and of temperature gradients in the rnedium. Also, this improved code allows one to consider non-uniform distributions of ash. 


\subsubsection{Overview}

In this research program, we have adopted the approach that by measuring fundamental properties (i.e, the complex refractive index, $m$ ) of the fly ash which participates in the radiation transfer, we can use well established theoretical pi inciples (Mie theory) to compute the radiative properties of dispersions of fly ash as found in coal combustors. With this approach one can understand the underlying principles that affect the radiative properties of an ash dispersion and more confidently predict how variations in the characteristics of the ash dispersion cause variations in its radiative properties.

An important criterion in this approach is that the flyash particles be spherical, homogeneous, and isotropic. Fortunately, fly ash particles are formed at high temperatures at which most of them are molten, leading primarily to spherical particles. Furthermore, one should expect that molten particles will be reasonably homogeneous and isotropic. On cooling, most fly ash particles form glassy spheres which are homogeneous and isotropic. Some ash particles form hollow shells (cenospheres) while others form as particles with "b:bbles" or voids, but most fly ash particles are well approximated as homogeneous isotropic spheres.

In the following sections we review some of the underlying principles that affect the radiative properties of $f y$ ash dispersions and report on progress that has been made during the past quarter.

\subsubsection{Scattering Theory}

Mie theory is the exact solution to Maxwell's equations for scattering and absorption by a homogeneous isotropic sphere. It is well established both theoretically and experimentally: but until the advent of modern computers, it was difficult to compute except in certain limits (e.g. Rayleigh theory for small particles, geometric and physical optics theory for large particles, and Rayleigh-Gans theory for weakly scattering particles). Today, calculations are routinely made for spherical particles of arbitrary size and refractive index.

Given the complex refractive index $m=n+i k$ at wavelength $\lambda$ for a particle with diameter $D$, the spectral scattering efficiency, $Q_{s . \lambda}$, the spectral absorption efficiency, $\mathrm{Q}_{\mathrm{a} . \lambda}$. and the spectral scattering phase function. $\Phi_{\lambda}(\theta)$ can be computed using established techniques [2]. The "efficiency" is defined as the total power being considered 
(e.g. scattered or absorbed power) divided by the power incident on the cross sectional area of the particle. For example, $Q_{a, \lambda}=$ total power absorbed/total power incident on cross section. The scattering phase function, $\Phi_{\lambda}(\theta)$, describes the angular distribution of scattered radiation, normalized so that $\Phi_{\lambda}=1$ for isotropic scattering.

It is helpful to understand the limiting cases before considering particles of arbitrary size. For very small particles, $x \equiv \pi D / \lambda \ll 1$, the scattering efficiency, $\mathrm{Q}_{\mathrm{s}, \lambda}$ is proportional to $x^{4}$ and the absorption efficiency, $Q_{\mathbf{a}, \lambda}$ is proportional to $x$. In this Rayleigh limit, particles scatter very littie (e.g. gas molecules typically have $x \ll 1$ ). The scattering rhase function, which describes the angular distribution of scattered light for randomly polarized incident radiation, approaches the limit

$$
\Phi_{\lambda}(\theta) \sim \frac{3}{4}\left(1+\cos ^{2} \theta\right) ; x \ll 1
$$

where $\theta$ is the angle measured from the forward direction.

In the large particle limit, $x \rightarrow \infty$. the extinction efficiency: $Q_{e, \lambda} \equiv Q_{s, \lambda}+Q_{a, \lambda}$, approaches the limiting value of two. For very weakly absorbing particles, $Q_{a, \lambda} \rightarrow 0$, we have $Q_{s, \lambda} \rightarrow 2$, where half of the scattering is due to refraction and reflection as described by geometric optics, and half of the scattering is due to diffraction as described by physical optics. For strongly absorbing particles. $Q_{a, \lambda} \rightarrow 1-Q_{r, \lambda}$ and $Q_{s, \lambda} \rightarrow 1+Q_{r, \lambda}$ where $Q_{r, \lambda}$ is the reflection efficiency:

The scattering by very large particles is strongly concentrated in the forward direction, and the scattering phase function is approximated in the forward direction by

$$
\Phi_{\lambda}(\theta) \sim x^{2}\left(\frac{2 J_{1}[x \sin \theta]}{x \sin \theta}\right)^{2} ; x \rightarrow \infty
$$

where $J_{1}$ is the Bessel function of the first kind of order one. Eq. (2) does not precisely prescribe the scattering by large particles in all directions, but does accurately predict that most of the light scattered from a large particle is in a narrow forward lobe.

Figure 2 shows a comparison of the scattering phase function computed using exact Mie theory with Eq. (2). For $x=10$ and $x=100$ the large particle approximation agrees qualitatively well. All curves show that $\Phi_{\lambda}(\theta)$ is approximately two orders of magnitude larger at $\theta=0$ than at the peak of the next lobe. 


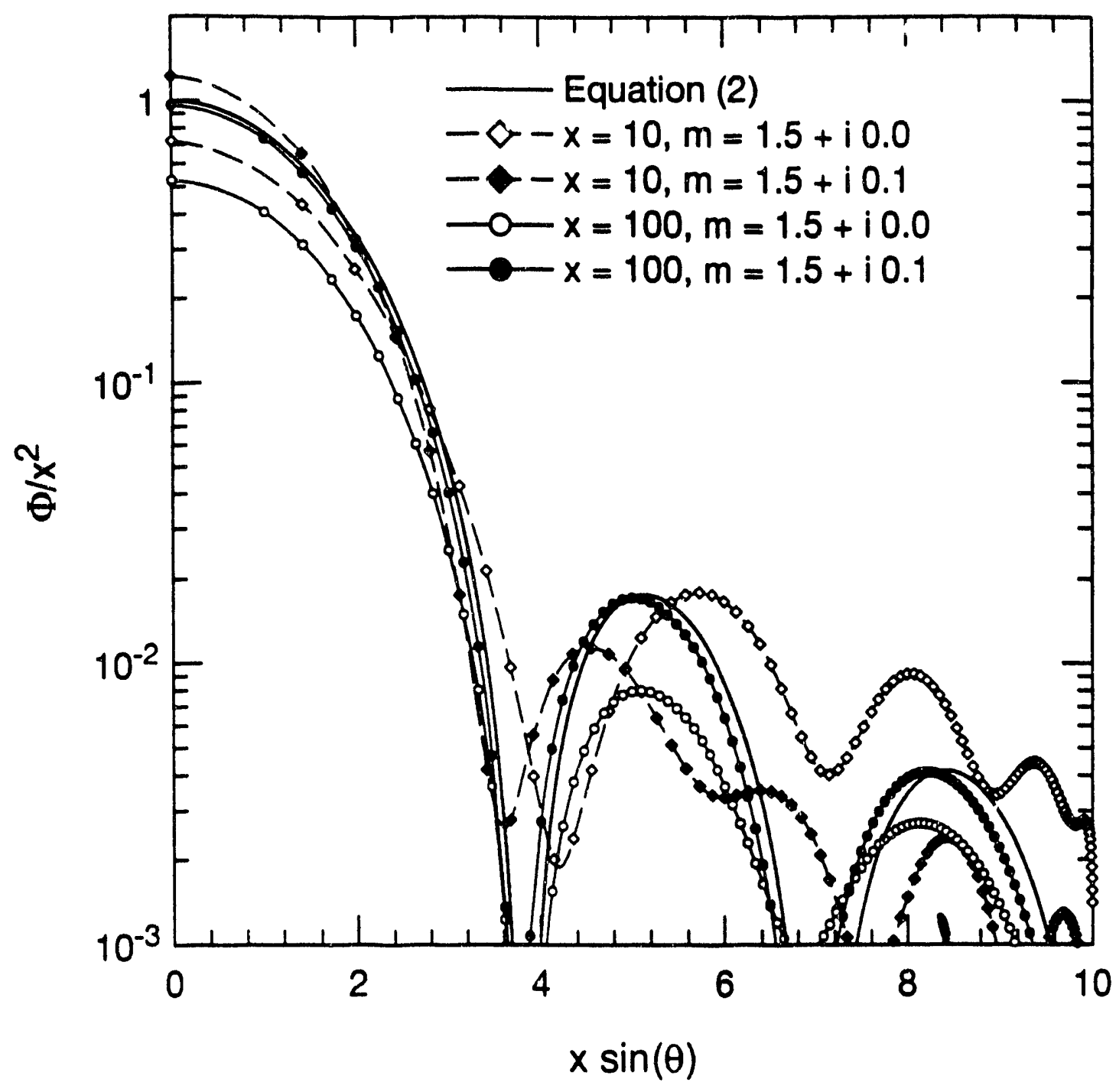

Figure 2: The scattering phase function for large size parameter

The width of the forward lobe is reasonably well predicted by Eq. (2) even for $x$ as small as 10 . The forward lobe spans an angle of approximately $\sin ^{-1}(4 / x)$ corresponding to angles of approximately $24^{\circ}$ and $2.3^{\circ}$ for $x=10$ and $x=100$ respectively. From this quick calculation we can conclude that radiation incident on large particles ( $x \geq 100$ or so) will either be absorbed or scattered into a direction nearly equal to the incident direction (i.e., negligible scattering).

\subsubsection{Average Radiative Properties}

Fly ash particles vary in diameter over several orders of magnitude and the composition varies from particle to particle. Before radiation heat transfer calculations can 
be made for real fly ash dispersions, average scattering and absorption coefficients must be computed.

Consider the spectral radiative transfer equation for a mono-dispersion of particles with diameter $\mathrm{D}$ and uniform composition $\mathrm{C}$; in a non-participating medium

$$
\frac{d I_{\lambda}}{d s}=-\left(\sigma_{\lambda, \mathrm{DC}}+\alpha_{\lambda, \mathrm{DC}}\right) I_{\lambda}+\alpha_{\lambda, \mathrm{DC}} I_{b, \lambda}(T)+\frac{\sigma_{\lambda, \mathrm{DC}}}{4 \pi} \int_{4 \pi} \boldsymbol{\Phi}_{\lambda, \mathrm{DC}}\left(\boldsymbol{\Omega} \cdot \boldsymbol{\Omega}^{\prime}\right) I_{\lambda}\left(\boldsymbol{\Omega}^{\prime}\right) \mathrm{d} \boldsymbol{\Omega}^{\prime}
$$

$I_{\lambda}$ is the spectral radiance at position $s$ in direction $\boldsymbol{\Omega}$. The scattering and absorption coefficients for particles with diameter $\mathrm{D}$ and composition $\mathrm{C}$ are given by

$$
\begin{aligned}
& \sigma_{\lambda, D C}=Q_{s, \lambda}(D, C) \pi \frac{D^{2}}{2} n \\
& \alpha_{\lambda, D C}=Q_{a, \lambda}(D, C) \pi \frac{D^{2}}{2} n
\end{aligned}
$$

where $n$ is the number of particles per unit volume and $G_{s, \lambda}(D, C)$ and $Q_{a, \lambda}(D, C)$ are the scattering and absorption efficiencies computed with Miie theory for $x=\pi \mathrm{D} / \lambda$ and $m=n+i k$ of composition $\mathrm{C}$.

If there is a continuous distribution of particles sizes, $f_{C}(D)$, with composition $C$, then the number of particles per unit volume with diameter $D$ within $d D$ becomes $N_{C} f_{C}(D) d D$ where $N_{C}$ is the total number of particles (of any size) with composition $C$ per unit volume and $f_{C} d D$ is the fraction of particles with diameter between $D$ and $(D+d D)$.

Replacing $n$ with its differential form in Eq. (4) and integrating Eq. (3) over all diameters, $\mathrm{D}$, yields the size distribution averaged equation of transfer for a polydispersion of particles with composition $\mathrm{C}$,

$$
\frac{d I_{\lambda}}{d s}=-\left(\sigma_{\lambda, \mathrm{C}}+\alpha_{\lambda, \mathrm{C}}\right) I_{\lambda}+\alpha_{\lambda, \mathrm{C}} I_{b, \lambda}(T)+\frac{\sigma_{\lambda, \mathrm{C}}}{4 \pi} \int_{4 \pi} \boldsymbol{\Phi}_{\lambda, \mathrm{C}}\left(\boldsymbol{\Omega} \cdot \boldsymbol{\Omega}^{\prime}\right) I_{\lambda}\left(\boldsymbol{\Omega}^{\prime}\right) \mathrm{d} \boldsymbol{\Omega}^{\prime}
$$

where the averaged coefficients are of the form:

$$
\begin{aligned}
& \sigma_{\lambda, C}=-\frac{1 C_{C} \pi}{4} \int_{0}^{\infty} Q_{s, \lambda}(D, C) D^{2} f(D) d D \\
& \alpha_{\lambda, C}=\frac{N_{\ulcorner}}{4} \int_{0}^{\infty} Q_{a, \lambda}(D, C) D^{2} f(D) d D .
\end{aligned}
$$

The size averaged scattering phase function is given by

$$
\Phi_{\lambda . C}(\theta)=\frac{\pi \AA}{4 \sigma_{\lambda . C}} \int_{0}^{\infty} \Phi_{\lambda . D C}(\theta) Q_{s . \lambda}(D, C) D^{2} f(D) d D
$$


Even though the compositions of fly ash particles vary considerably from particle to particle, the complex refractive index varies much less. To good approximation one can replace the continuous composition distribution with a discrete one. Thus we may divide the fly ash dispersion into a finite number of classes where each class, C, corresponds to a range of compositions for which the complex refractive index, $m_{\mathrm{C}}$, and size distribution, $f_{C}(D)$ are approximately constant. The radiative properties for the average composition are obtained by summing over all compositions classes. The scattering and absorption coefficients for the dispersion become

$$
\begin{gathered}
\sigma_{\lambda}=\frac{\sum_{\text {all } C} A_{C} \sigma_{\lambda, C}}{\sum_{\text {all } C} A_{C}} \\
\alpha_{\lambda}=\frac{\sum_{\text {all } C} A_{C} \alpha_{\lambda, C}}{\sum_{\text {all } C} A_{C}}
\end{gathered}
$$

where $A_{C}$ is the fraction of the total projected area of all the particles due to particles of composition $C$. and is given by

$$
A_{C}=N_{C} \int_{0}^{\infty} \frac{\pi D^{2}}{4} f_{C}(D) d D .
$$

The scattering phase function for the dispersion becomes

$$
\Phi_{\lambda}(\theta)=\frac{\sum_{\text {all } C} A_{C} \sigma_{\lambda, C} \Phi_{\lambda, C}}{\sum_{\text {all } ~} A_{C} \sigma_{\lambda, C}} .
$$

These relations demonstrate the procedure one must follow to obtain averaged radiative transport properties for a fly ash dispersion. The calculations require many Mie scattering computations and a knowledge of the size and composition distribution of the fly ash dispersion. The Mie calculations are very time consuming, but have been successfully implemented on an IBM PC. Results of the calculations will be presented when data from Task 1 and Task 2 are available.

\subsubsection{Radiation Heat Transfer Calculations}

In a real pulverized coal combustor. the fly ash is not uniformly distributed and is not at a uniform temperature. Furthermore. the composition and size distributions will vary with position in the combustor. since smaller and lighter particles will follow 
the flow field more closely than larger and heavier particles. Therefore, the average properties will vary with position throughout the combustor in a complicated way. To account for this spatial variation and study its effects on heat transfer, a new computer code has been developed that ailows for spatial variations in temperature and radiative properties. In this section the solution technique is presented for a one-dimension planar medium. The method may easily be extended to higher dimensions.

\section{The Radiative Transport Equation}

The radiative transport equation for a one dimensional planar medium is [3]

$$
\begin{aligned}
\mu \frac{d I(x, \mu)}{d x}= & -[\sigma(x)+\alpha(x)] I(x, \mu)+\alpha(x) I_{b}[T(x)] \\
& +\frac{\sigma(x)}{4 \pi} \int_{0}^{2 \pi} \int_{-1}^{j} \Phi\left(\mu_{0}\right) I\left(x, \mu^{\prime}\right) d \mu^{\prime} d \phi
\end{aligned}
$$

where $x$ is the coordinate normal to the infinite planar slab, $\mu$ is the cosine of the angle measured from the positive $x$-direction, and $T(x)$ is the temperature distribution in the medium. The radiance, $I(x, \mu)$, and all the radiative properties are spectral quantities but the subscript $\lambda$ is dropped for brevity. The scattering phase function, $\Phi\left(\mu_{0}\right)$ depends only on the angle between incident and scattered radiation and is given by

$$
\mu_{0}=\boldsymbol{\Omega} \cdot \boldsymbol{\Omega}^{\prime}=\mu \mu^{\prime}-\sqrt{1-\mu^{\prime 2}} \sqrt{1-\mu^{2}} \cos \left(\phi-\phi^{\prime}\right),
$$

where the azimuthal angle $\phi$ lies in the range $0 \leq \phi \leq 2 \pi$.

It is convenient to introduce the dimensionless optical depth. $\tau$,

$$
\tau \equiv \int_{0}^{x}(\alpha+\sigma) d x^{\prime}
$$

as the new independent space variable and the dimensionless scattering albedo

$$
\omega(\tau) \equiv \frac{\sigma(\tau)}{\alpha(\tau)+\sigma(\tau)} .
$$

For a slab with thickness $\ell$ the total optical depth is simply $\tau=\int_{0}^{\ell}(a+\sigma) d x$. With this change of variables the radiation transport equation, Eq. ( 8 ), becomes

$$
\begin{aligned}
\mu \frac{\partial I(\tau, \mu)}{\partial \tau} & =-I(\tau, \mu)+S(\tau, \mu) \\
S(\tau, \mu) & \equiv\left[1-\omega^{\prime}(\tau)\right] I_{b}[T(\tau)]+\frac{\omega(\tau)}{4 \pi} \int_{0}^{2 \pi} \int_{-1}^{1} \Phi\left(\mu_{0}\right) I\left(\tau, \mu^{\prime}\right) d \mu^{\prime} d \phi
\end{aligned}
$$


Solutions of equation (12) are obtained using the discrete ordinates method [4-5]. In that method, Eq. (12) is approximated by evaluation of the direction cosine, $\mu$, at a finite number of discrete directions, $\mu_{m}$, and integrals over $\mu$ become finite summations. For example, an integral is approximated by the quadrature formula

$$
\int_{-1}^{1} f(\mu) d \mu=\sum_{m=1}^{M} f\left(\mu_{m}\right) w_{m}
$$

where the ordinate, $\mu$, has been divided into $M$ discrete values $\mu_{m}$ with $w_{m}$ being the weight associated with $\mu_{m}$.

Quadrature Formula

An important aspect of the discrete ordinates method is selection of the directions, $\mu_{m}$. It is useful to understand quadrature formulae so that "good" choices for $\mu_{m}$ are made.

Following the development from Chandrasekhar [4], consider the general integral

$$
I=\int_{a}^{b} f(x) w(x) d x
$$

where $f(x)$ is some general function and $w(x)$ is a weight function. The goal is to construct a suitable quadrature formula (approximate summation) which closely approximates $I$ using a finite number, $M$, of discrete abscissas, $x_{j}$.

Letting $x_{j}$ be one of the set $\left(x_{1}, x_{2}, \ldots, x_{M}\right)$, where $a \leq x_{j} \leq b$, we construct a polynomial using Lagrange's formula

$$
\phi(x)=\sum_{j=1}^{M} f\left(x_{j}\right) \frac{F(x)}{\left(x-x_{j}\right) F^{\prime}\left(x_{j}\right)}
$$

where

$$
F(x)=\prod_{j=1}^{M}\left(x-x_{j}\right) \text { and } F^{\prime}\left(x_{j}\right)=\prod_{\substack{i=1 \\ i \neq j}}^{M}\left(x_{j}-x_{i}\right)
$$

From this formula is is clear that $\phi(x)=f(x)$ at the quadrature points $x_{j}$. Moreover, if $f(x)$ is a polynomial of order $M-1$ or less, $\phi(x)$ is exactly equal to $f(x)$ for all $x$.

Substituting the polynomial approximation. $\phi(x)$, for the function $f(x)$ in the integral $I$ gives

$$
I \simeq \int_{a}^{b} O(x) u^{\prime}(x) d x=\sum_{j=1}^{M} \frac{f\left(x_{j}\right)}{F^{\prime}\left(x_{j}\right)} \int_{a}^{b} \frac{F(x) w(x)}{\left(x-x_{j}\right)} d x
$$


or, by defining $a_{j}$ as

$$
a_{j} \equiv \frac{1}{F^{\prime}\left(x_{j}\right)} \int_{a}^{b} \frac{F(x) w(x)}{x-x_{j}} d x
$$

we obtain

$$
I \simeq \sum_{j=1}^{M} a_{j} f\left(x_{j}\right)
$$

The error introduced by approximating Eq. ( 13) with Eq. (16) is

$$
\epsilon_{M}=I-\sum_{j=1}^{M} a_{j} f\left(x_{j}\right) .
$$

Since $\phi(x)$ was an $M^{\text {th }}$ order polynomial passing exactly through $M$ points of the function $f(x)$, the error, $\epsilon_{M}$, vanishes if $f(x)$ is a polynomial of order $M-1$ or less; so long the $M x_{j}$ 's are unique, any $x_{j}$ 's will do. The question arises: can the $x_{j}$ be chosen in a "smart" way to make the error vanish for cases when $f(x)$ is a polynomial of order higher than $M-1$ ? Carl Friedrich Gauss showed that $x_{j}$ can be chosen in such a way.

Consider the case when $f(x)$ is a polynomial of order $2 M-1$. Define the moments of the weight function $w(x)$ as

$$
\alpha_{\ell}=\int_{a}^{b} x^{\ell} w(x) d x . \quad ; \quad(\ell=0,1, \ldots, 2 M-1)
$$

For the approximate quadrature formula to be exact, the moments must satisfy the equations

$$
\alpha_{\ell}=\sum_{j=1}^{M} a_{j} x_{j}^{\ell} \quad ; \quad(\ell=0,1, \ldots, 2 M-1)
$$

which gives $2 M$ equations for the $2 M$ unknowns $\left(a_{1}, \ldots, a_{M}\right)$ and $\left(x_{1}, \ldots, x_{M}\right)$.

Chandrasekhar [4] carefully outlines a method for finding the $a_{j}$ and $x$, using Eq. ( 19), thus demonstrating that one cars construct an $M$ point quadrature formula that will exactly evaluate integrals of weighted polynomials of order $2 M-1$. This result is very important and not very intuitive a priori.

Now that it is established that there are "good" choices for $x$, reconsider the original approximating polynomial. $\phi(x)$, which is a polynomial of order $M$. Recall 
that $\phi(x)$ is equal to $f(x)$ at the $x$-values $\left(x_{1}, \ldots, x_{M}\right)$ and the function $F(x)=$ $\prod_{j=1}^{M}\left(x-x_{j}\right)$ is also a polynomial of order $M$. If $f(x)$ is a polynomial of order $2 M-1$ it can be written as

$$
f(x)=\phi(x)+F(x) \sum_{\ell=0}^{M-1} q_{\ell} x^{\ell}
$$

where $q_{\ell}$ are known constants for a given polynomial $f(x)$. Using this expression for $f(x)$ the error, Eq. ( 17), of the quadrature formula is

$$
\begin{aligned}
\epsilon_{M}= & \int_{a}^{b} f(x) w(x) d x-\sum_{j=1}^{M} a_{j} f\left(x_{j}\right) \\
= & \int_{a}^{b} \phi(x) w(x) d x+\int_{a}^{b} F(x)\left(\sum_{\ell=0}^{M-1} q_{\ell} x^{\ell}\right) w(x) d x \\
& \quad-\int_{a}^{b} \phi(x) w(x) d x \\
= & \sum_{\ell=0}^{M-1} q_{\ell} \int_{a}^{b} F(x) w(x) x^{\ell}
\end{aligned}
$$

Since all the $q_{\ell}$ are not necessarily zero, the error, $\epsilon_{\mathcal{M}}$, vanishes only if

$$
\int_{a}^{b} F(x) w(x) x^{\ell}=0 \quad, \quad(\ell=0, \ldots, M-1) .
$$

This condition determines $F(x)$ and the ordinates $x_{j}$, which are simply found from $F\left(x_{j}\right)=0$. Once $x_{j}$ are known, the weights, $a_{j}$ are determined from Eq. (15).

Consider the special case of $w=1$ and the limits $a=-1, b=1$. For this special case the integral, $I$, is defined as

$$
I \equiv \int_{-1}^{1} f(\mu) d \mu
$$

and the condition determining the function $F(\mu)$ becomes

$$
\int_{-1}^{1} F(\mu) \mu^{\ell}=0 \quad, \quad(\ell=0, \ldots, M-1) .
$$

A function $F(\mu)$ which satisfies Eq. ( 22) for all $\ell<M$ is the Legendre polynomial $P_{M}(\mu)$. Therefore. the choice of $\mu$, as the roots of the Legendre polynomial $P_{. M}(\mu)=0$ yields a quadrature formula with $M$ terms that is capable of exactly evaluating integrals 
of polynomials of order $2 M-1$. The weight factors $w_{j}$ (previously termed $a_{j}$ ) are determined from Eq. ( 15 ) to be

$$
w_{j}=\frac{1}{P_{M}^{\prime}\left(\mu_{j}\right)} \int_{-1}^{1} \frac{P_{M}(\mu)}{\mu-\mu_{j}} d \mu .
$$

This choice of $\mu_{j}$ and $w_{j}$ is known as the Gauss quadrature and the "Gauss quadrature formula" is simply

$$
\int_{-1}^{1} f(\mu) d \mu \simeq \sum_{j=1}^{M} f\left(\mu_{j}\right) w_{j}
$$

Press, et. al. [6] discusses algorithms that quickly compute $\mu_{j}$ and $w_{j}$ for a given order $M$.

\section{The Discrete Ordinates Method}

The Gauss quadrature formula developed in the previous section is used to approximate the radiative transport equation, Eq. ( 12), as $M$ equations for the "discrete ordinate" directions $\left(\mu_{1}, \ldots, \mu_{M}\right)$. Integrals in $\mu$ are replaced by quadrature summations and the resulting system of equations is

$$
\mu_{m} \frac{d I^{m}}{d \tau}+I^{m}=S^{m} \quad(m=1,2, \ldots, M)
$$

where

$$
S^{m} \equiv S\left(\tau, \mu_{m}\right) \simeq(1-\omega) I_{b}+\frac{\omega}{2} \sum_{j=1}^{M} \Phi\left(\mu_{m}, \mu_{j}\right) I^{j} w_{j}
$$

and

$$
\Phi\left(\mu_{m}, \mu_{j}\right) \equiv \frac{1}{2 \pi} \int_{0}^{2 \pi} \Phi\left(\mu_{0}\right) d \phi .
$$

The scattering albedo, $\omega$, and Plank blackbody function, $I_{b}$, are both general functions of position $\tau$, as could also be the scattering phase function $\Phi\left(\mu_{m}, \mu_{j}\right)$.

The scattering phase function, $\Phi\left(\mu_{m}, \mu_{3}\right)$, is evaluated by expanding $\Phi\left(\mu_{0}\right)$ as a series of Legendre polynomials,

$$
\Phi\left(\mu_{0}\right)=\sum_{i=0}^{\infty} A_{i} P_{i}\left(\mu_{0}\right), \quad A_{0}=1
$$

where $\mu$ is in the range $-1 \leq \mu \leq 1$. Using the orthogonality relation for $P_{m}(\mu)$

$$
\int_{-1}^{1} P_{i}(\mu) P_{j}(\mu) d \mu=\frac{2}{2 j+1} \delta_{i j}
$$


where $\delta_{i j}=0$ if $i \neq j$ and 1 if $i=j$, one can determine the expansion coefficients, $A_{j}$, from the relation

$$
A_{j}=\frac{2 j+1}{2} \int_{-1}^{1} \Phi(\mu) P_{j}(\mu) d \mu \text {. }
$$

Once the $A_{j}$ are determined for a given $\Phi(\mu)$, is remains to compute $\Phi\left(\mu, \mu^{\prime}\right)$ from Eq. ( $25 \mathrm{c}$ ). In practice the infinite Legendre series expansion is truncated after a finite number of terms, $N$, so that

$$
\Phi\left(\mu_{m}, \mu_{j}\right) \simeq \sum_{n=0}^{N} A_{n} P_{n}\left(\mu_{m}\right) P_{n}\left(\mu_{j}\right) .
$$

Mie calculations indicate that for large particles, choosing $N$ as approximately twice the particle size parameter, $x$, is sufficient to resolve the phase function with adequate accuracy. The Legendre polynomials are computed using upward recurrence from the relation

$$
P_{n}(\mu)=\frac{(2 n-1) \mu P_{n-1}-(n-1) P_{n-2}}{n} ; \quad P_{1}(\mu)=\mu ; \quad P_{0}(\mu)=1 .
$$

The next step in the discrete ordinates method is to finite difference the spatial derivative using a non-uniform grid in $\tau$. Divide the slab into $L$ planar layers with $L+1$ surfaces at $\left(\tau_{0}, \tau_{1}, \ldots, \tau_{L}\right)$. Using central differences $\left(2^{\text {nd }}\right.$ order accurate $)$ about $i+\frac{1}{2}$ one can approximate Eq. ( $\left.25 \mathrm{a}\right)$ as

$$
\mu_{m}\left(\frac{I_{i+1}^{m}-I_{i}^{m}}{\Delta \tau_{i}}\right)+I_{i+\frac{1}{2}}^{m}=S_{i+\frac{1}{2}}^{m}
$$

where

$$
\Delta \tau_{i} \equiv \tau_{i+1}-\tau_{i} \quad \text { and } \quad I_{i}^{m} \equiv I\left(\tau_{i}, \mu_{m}\right) .
$$

The values at half intervals $\left(i+\frac{1}{2}\right)$ are evaluated by averaging on the two interfaces bounding the interval, i.e.,

$$
I_{i+\frac{1}{2}}^{m}=\frac{1}{2}\left(I_{i+1}^{m}+I_{i}^{m}\right) \quad S_{i+\frac{1}{2}}^{m}=\frac{1}{2}\left(S_{i+1}^{m}+S_{i}^{m}\right) .
$$

Finally, rearranging terms in Eq. (29) and substituting Eq. ( 30 ), the difference formula is written as

$$
I_{i+1}^{m}=\left(\frac{\frac{\mu_{m}}{\frac{\Delta \tau_{i}}{\mu_{m}}-\frac{1}{2}}}{\frac{\mu_{m}}{\Delta \tau_{i}}+\frac{1}{2}}\right) I_{i}^{m}+\frac{\frac{1}{2}\left(S_{i+1}^{m}+S_{i}^{m}\right)}{\frac{\mu_{m}}{\Delta \tau_{i}}+\frac{1}{2}} \quad\left(\mu_{m}>0\right)
$$

This difference equation is evaluated only in the forward direction. $\left(\mu_{m}>0\right)$. by starting with boundary values at $\tau=\tau_{0}$ and stepping forward in $\tau$. A similar equation 
is formulated for stepping in the backward direction, $\mu_{m}<0$. Following a procedure similar to that above, one can write

$$
I_{i-1}^{m}=\left(\frac{\frac{\mu_{m}}{\Delta T_{i-1}}+\frac{1}{2}}{\frac{\mu_{m}}{\Delta T_{i-1}}-\frac{1}{2}}\right) I_{i}^{m}-\frac{\frac{1}{2}\left(S_{i}^{m}+S_{i-1}^{m}\right)}{\frac{\mu_{m}}{\Delta \tau_{i-1}}-\frac{1}{2}} . \quad\left(\mu_{m}<0\right)
$$

Note that $S_{i}^{m}$ depends on the radiance, $I_{i}^{j}$ at $\tau_{i}$ in all directions $\mu_{j}(j=1, \ldots, M)$ through Eq. ( 25b). However, one can overcome this complication by using iteration. That is, we assume initially that the $S_{i}^{m}$ are known for all $m$ and $i$, then compute the radiance $I_{j}^{m}$ using Eq. (31). Next, we recompute $S_{j}^{m}$ using the newly acquired radiance, $I_{j}^{m}$. This iteration is repeated until subsequent calculations of $I_{j}^{m}$ produce negligible change.

\section{Boundary Conditions}

For unique solutions to exist, one must specify the boundary conditions at $\tau=0$ and $\tau=\tau_{0}$. Since the analysis thus far has been restricted to azimuthally symmetric radiation in a plane slab (1-D), azimuthally symmetric boundary conditions are also used. Consider the boundaries to be diffusely emitting, partially diffuse reflecting, and partially specular reflecting. Thus

$$
\begin{array}{cl}
I(0, \mu)=\epsilon_{0} I_{b}\left(T_{0}\right)-2 \rho_{0}^{d} \int_{-1}^{0} I\left(0, \mu^{\prime}\right) \mu^{\prime} d \mu^{\prime}+\rho_{0}^{s} I(0,-\mu) ; & (\mu>0) \\
I\left(\tau_{0}, \mu\right)=\epsilon_{L} I_{b}\left(T_{1}\right)+2 \rho_{L}^{d} \int_{0}^{1} I\left(\tau_{0}, \mu^{\prime}\right) \mu^{\prime} d \mu^{\prime}+\rho_{L}^{s} I\left(\tau_{0},-\mu\right) ; & (\mu<0)
\end{array}
$$

where $\epsilon$ is the diffuse emissivity, $\rho^{d}$ is the diffuse reflectivity, $\rho^{s}$ is the specular reflectivity, and subscripts " 0 " and " $L$ " denote the boundaries at $\tau=0$ and $\tau=\tau_{0}$ respectively.

The integrals in the boundary conditions are replaced with Gauss quadrature formulas to give

$$
\begin{array}{cc}
I_{0}^{m}=\epsilon_{0} I_{b 0}-2 \rho_{0}^{d} \sum_{\substack{j \\
\mu_{j}<0}} I_{0}^{j} \mu_{j} w_{j}+\rho_{0}^{s} I_{0}\left(-\mu_{m}\right) & \left(\mu_{m}>0\right) \\
I_{L}^{m}=\epsilon_{L} I_{b L}-2 \rho_{L}^{d} \sum_{\substack{j \\
\mu_{j}>0}} I_{L}^{j} \mu_{j} w_{j}+\rho_{L}^{s} I_{L}\left(-\mu_{m}\right) & \left(\mu_{m}<0\right)
\end{array}
$$

Note that the radiation incident on the slab boundaries is dependent on the emergent radiance if the boundaries are reflecting. However, by iterating as described for $S_{i}^{m}$, the solution can be obtained without solving any time consuming matrix equations for the boundary intensities. 
Convergence Criterion Iteration is used as discussed above to avoid the implicit dependence of the source function $S_{i}^{m}$ and the boundary conditions on the radiance, $I_{i}^{m}$. Convergence of the iterative process is presumed when the heat flux at each optical depth, $\tau_{i}$, changes from one iteration to the next by less than some prescribed small error. The heat flux through the slab can be computed at each optical depth, $\tau_{i}$, and for each iteration using the quadrature approximation

$$
Q_{i} \simeq 2 \pi \sum_{m=0}^{M} I_{i}^{m} \mu_{m} w_{m} .
$$

Solution Procedure During the past quarter, the formulation presented above was implemented in a computer program. The algorithm is relatively simple, since all equations are solved explicitly and iteration is used to find the solution. The following summary outlines the necessary steps of the computation:

1. Input slat thickness and number of divisions $L$, number of ordinates $M$, absorption coefficient $\alpha(x)$, scattering coefficient $\sigma(x)$, temperature distribution $T(x)$, Legendre coefficients $\left(A_{0}, \ldots, A_{N}\right)$, and boundary conditions.

2. Compute optical depths $\left(\tau_{0}, \ldots, \tau_{L}\right)$, albedo $\left(\omega_{0}, \ldots, \omega_{L}\right)$, ordinates $\left(\mu_{1}, \ldots, \mu_{M}\right)$ and weights $\left(u_{1}, \ldots, w_{M}\right)$, and scattering phase function $\Phi\left(\mu_{i}, \mu_{j}\right)$.

3. Set initial values for $I_{i}^{m} . S_{i}^{m}$. and $Q_{i}^{m}$.

4. Compute $I_{i}^{m}$ from Eq. ( 31$)$.

5. Using new $I_{i}^{m}$ from step 4. compute $S_{i}^{m}$ from Eq. (25b).

6. Compute boundary intensities from Eq. (33).

7. Compute $Q_{i}$ at all $\tau_{i}$ and compare with previous $Q_{i}$. If change is greater than some error, $\epsilon$, repeat steps $4-7$.

8. Print results. 


\subsubsection{Preliminary Results and Code Evaluation}

Our immediate goal is to evaluate the performance and limitations of the computational procedure outlined above. A number of computations were made to check the validity and accuracy of the codes. In all cases the codes produced the expected results when compared to exact calculations for isotropic scattering, using previously developed codes [1], and to published [3] results for anisotropic scattering. No significant accuracy or convergence problems were encountered.

In order to simplify this evaluation stage of code development, all the calculations are for monodispersions of single-composition particles at a single wavelength. We assume that the medium is composed of single-sized spheres with complex refractive index, $m=1.5+i 10^{-4}$. The size of the spheres, the slab thickness or particle number density, and the absorption coefficient of the surrounding medium is varied to allow investigation of the effect of anisotropic scattering, optical depth, and scattering albedo on the heat flux and emergent radiance.

The Scattering Phase Function The scattering rhase function was computed for particles sizes ranging from $x=0.5$ to $x=100$. The Legendre expansion coefficients, $A_{j}$, were computed using Eq. ( 27$)$ and Mie scattering theory to determine $\Phi(\mu)$. Table 3 shows some typical sets of expansion coefficients for particle size parameters $x=0.5$, $x=9$, and $x=36$. The series was truncated when the coefficients became smaller than $10^{-3}$, which generally corresponded to two or three times $x$ for large $x$.

When $x$ is large. the scattering phase function is very complicated with approximately $x$ scattering lobes. Therefore, many terms in a Legendre expansion are required to accurately fit the phase function. However, if the scattering phase function requires $N$ terms to be accurately modeled, then the discrete ordinates method will require at least $M=(N+1) / 2$ ordinates (directions) if accurate integrations of the phase function are to be performed (recall that the Gauss quadrature integration rule can exactly integrate polynomials of order $2 M-1$ using only $M$ abscissas). If scattering is very important $(\omega \rightarrow 1)$, as it is in fly ash dispersions [1]. then this problem is considerably more troublesome.

Figure 3 show's the effect of choosing too few ordinate directions on the transmittance, $0 \leq \mathrm{T} \leq 1$. of a plane slab of particles of size $x=50$. The limiting value at large $M$ is correct, but when scattering is important $(\omega=0.9)$ the results are only reasonable 
Table 3: Legendre Coefficients $A_{j}$ for $\Phi(\mu)=\sum_{j} A_{j} P_{j}(\mu)$ for single spheres with complex refractive index $m=1.5+i 10^{-4}$

\begin{tabular}{|c|c|c|c|c|c|c|c|}
\hline$j$ & & $A_{j}$ & & $j$ & $A_{j}$ & $j$ & $A_{j}$ \\
\hline & $x=0.5$ & $x=9$ & $x=36$ & \multicolumn{4}{|c|}{$x=36$ cont $\ldots$} \\
\hline 0 & 1.00000 & 1.00000 & 1.00000 & 28 & 17.92965 & 56 & 7.37375 \\
\hline 1 & 0.14660 & 1.94672 & 2.43076 & 29 & 17.83307 & 57 & 7.65716 \\
\hline 2 & 0.50398 & 2.99090 & 3.64363 & 30 & 17.84446 & 58 & 7.43930 \\
\hline 3 & 0.02760 & 2.95185 & 4.09975 & 31 & 17.74673 & 59 & 7.40488 \\
\hline 4 & 0.00064 & 3.72708 & 5.11313 & 32 & 17.61028 & 60 & 6.67121 \\
\hline 5 & & 3.73798 & 5.56431 & 33 & 17.54370 & 61 & 6.03762 \\
\hline 6 & & 4.23106 & 6.59476 & 34 & 17.23547 & 62 & 4.60609 \\
\hline 7 & & 4.07042 & 7.26245 & 35 & 17.17773 & 63 & 3.68276 \\
\hline 8 & & 3.91514 & 8.26331 & 36 & 16.71310 & 64 & 2.99628 \\
\hline 9 & & 3.59674 & 8.97579 & 37 & 16.62407 & 65 & 2.96285 \\
\hline 10 & & 2.93476 & 9.65623 & 38 & 16.07435 & 66 & 2.91504 \\
\hline 11 & & 2.43094 & 10.32124 & 39 & 15.90400 & 67 & 2.47309 \\
\hline 12 & & 1.76028 & 10.92715 & 40 & 15.34633 & 68 & 2.28324 \\
\hline 13 & & 1.24217 & 11.54660 & 41 & 15.06051 & 69 & 1.60334 \\
\hline 14 & & 1.08177 & 12.12031 & 42 & 14.46099 & 70 & 1.44967 \\
\hline 15 & & 0.92934 & 12.76528 & 43 & 14.01492 & 71 & 0.44679 \\
\hline 16 & & 1.19935 & 13.45026 & 44 & 13.28404 & 72 & 0.30244 \\
\hline 17 & & 0.91896 & 14.04943 & 45 & 12.69559 & 73 & -0.02195 \\
\hline 18 & & 1.13655 & 14.76339 & 46 & 11.81117 & 74 & 0.25306 \\
\hline 19 & & 0.21873 & 15.28887 & $4 i$ & 11.20362 & 75 & 0.07880 \\
\hline 20 & & 0.82504 & 15.92009 & 48 & 10.29457 & 76 & 0.05265 \\
\hline 21 & & $-0.0047 \pi$ & 16.33683 & 49 & 9.78790 & 77 & -0.07277 \\
\hline 22 & & 0.00940 & 16.84643 & 50 & 8.93928 & 78 & 0.02366 \\
\hline 23 & & 0.00228 & 17.11450 & 51 & 8.47910 & 79 & 0.00478 \\
\hline 24 & & 0.00042 & 17.48682 & 52 & 7.81775 & 80 & 0.00333 \\
\hline 25 & & & 17.59325 & 53 & 7.55318 & 81 & -0.00447 \\
\hline 26 & & & 17.83077 & 54 & 7.26440 & 82 & 0.00107 \\
\hline 27 & & & 17.80505 & 55 & 7.43740 & 83 & 0.00013 \\
\hline
\end{tabular}

for the two points $M=64$ and $M=128$. Figure 3 also shows that if scattering is not very important $(\omega=0.1)$ then an $M$ as small as 16 gives reasonably accurate results.

Scaling Anisotropic Scattering It is now evident why an alternative to solving the anisotropic equations is desirable. Even though it is plausible to use very large $M$ in the one-dimensional radiative transport problem, solutions for two or three dimensional geometries would require tremendous amounts of computer memory and time. One solution is to model the scattering phase function as partially isotropically scattering with the remaining scattered radiation in a forward-directed delta function, i.e.,

$$
\Phi(\mu) \simeq(1-g)+2 g \delta(\mu-1)
$$

where $g$ is the asymmetry parameter. the ratio of the forward scattered light to the backward scattered light. 


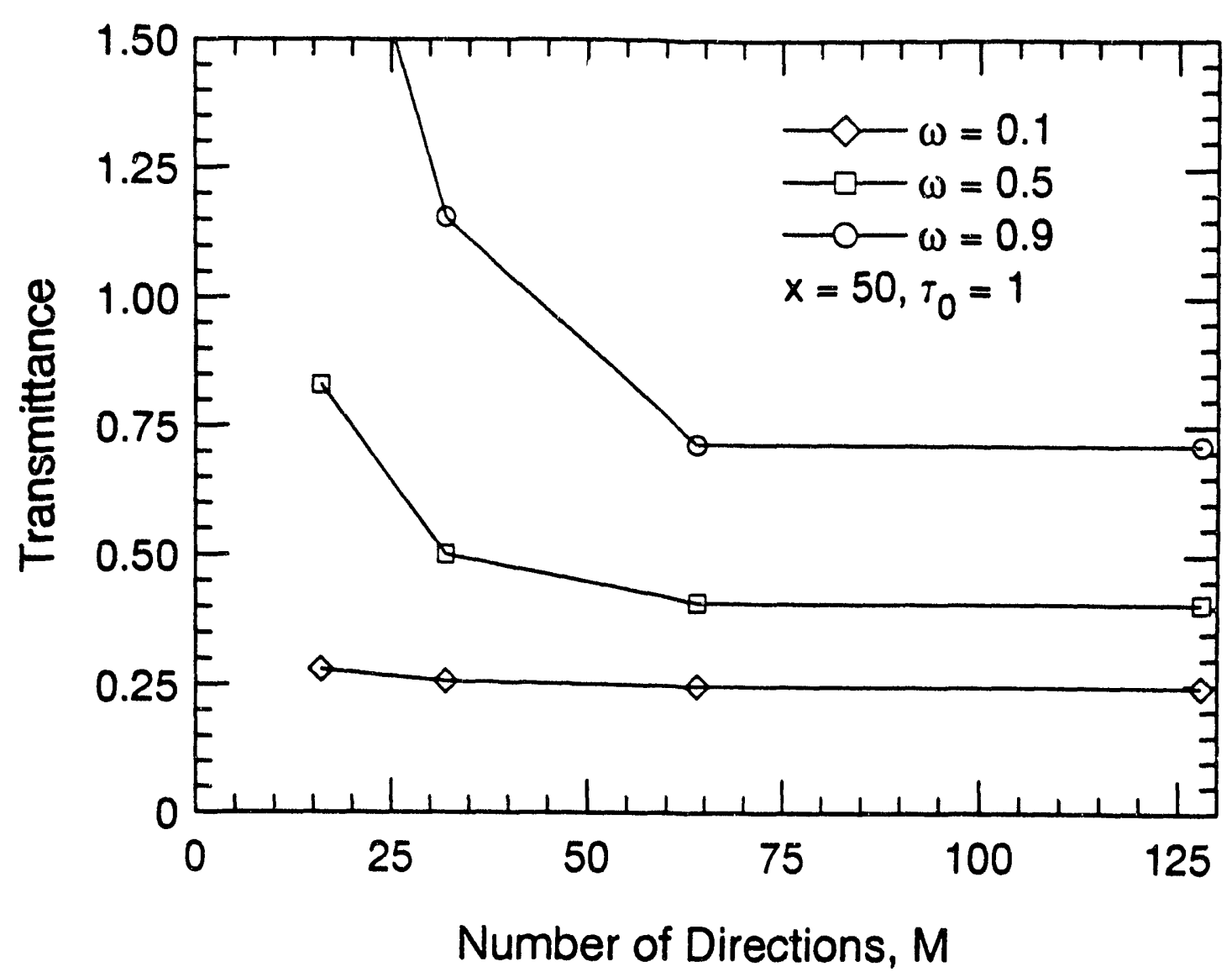

Figure 3: The transmittance of a slab versus the number of ordinates

By substituting Eq. ( 35 ) into the radiative transport equation, the forward directed portion of the scattering phase function vanishes and we are left with an isotropically scattering problem with an effective scattering coefficient

$$
\sigma^{\prime}=(1-g) \sigma,
$$

which translates into an effective optical depth, $\tau_{0}^{\prime}$, and effective scattering albedo, $\omega$, given by

$$
\begin{gathered}
\tau_{0}^{\prime}=(1-g \omega) \tau_{0} \\
\omega^{\prime}=(1-g) \omega /(1-g \omega) .
\end{gathered}
$$

The asymmetry parameter, $g$, is 1 for purely forward scattering, 0 for symmetric scattering (e.g. Rayleigh scattering), and - 1 for purely backward scattering. For compositions similar to most fly ash particles. the asymmetry parameter is generally greater than zero, i.e., the scattering is predominantly forward. The asymmetry parameter is 
defined as the first moment of the scattering phase functions and is therefore related to the Legendre expansion coefficient $A_{1}$ by

$$
g=\frac{1}{3} A_{1}
$$

Lee and Buckius [7] and Gupta et al. [8] have shown that scaling anisotropic scattering using this model scattering phase function leads to good accuracy for hemispherical quantities such as heat flux. To verify this scaling rule and also to check the correctness of the new computer code, the hemispherical transmittance and reflectance was computed for a range of particle sizes, $x$, with complex refractive index $m=1.5+i 10^{-4}$.

Table 4: Asymmetry Parameter, $g$, for spheres of various size with complex refractive index $m=1.5+i 10^{-4}$

\begin{tabular}{|c|c|c|c|}
\hline$x$ & $g$ & $x$ & $g$ \\
\hline 0.5 & 0.0489 & 16.0 & 0.7452 \\
1.0 & 0.1990 & 18.0 & 0.7890 \\
1.5 & 0.4998 & 20.0 & 0.7123 \\
2.0 & 0.6260 & 24.0 & 0.7795 \\
3.0 & 0.7344 & 28.0 & 0.7762 \\
4.0 & 0.7503 & 32.0 & 0.7732 \\
5.0 & 0.7076 & 36.0 & 0.8103 \\
6.0 & 0.6445 & 40.0 & 0.7880 \\
7.0 & 0.5196 & 50.0 & 0.8016 \\
8.0 & 0.4539 & 60.0 & 0.8045 \\
9.0 & 0.6489 & 70.0 & 0.8082 \\
10.0 & 0.7435 & 80.0 & 0.8196 \\
12.0 & 0.7886 & 90.0 & 0.8088 \\
14.0 & 0.7081 & 100.0 & 0.8224 \\
\hline
\end{tabular}

Table 4 shows the $x$-values for which calculations were performed and their corresponding asymmetry parameters, $g$. Notice that the asymmetry parameter increases from approximately 0.05 at $x=0.5$ to a maximum value of 0.82 at $x=100$. The general trend is for $g$ to increase with increasing $x$ as the forward scattered lobe becomes more pronounced. However, owing to the complicated nature of Mie scattering, the curve is not strictly monotonic. It is beyond the scope of this discussion to fully explain this phenomenon. but in large particles it can be explained by considering the interference between the forward diffracted light and the forward refracted light (the light transmitted through the particle). Small particles require a more complicated explanation since refraction is not very meaningful for small $x$.

Figures $4-6$ show the hemispherical emittance versus particle size, $x$, for various optical depths. $\tau_{0}$. and scattering albedos. $\omega^{\prime}$. The results for anisotropic scattering. 


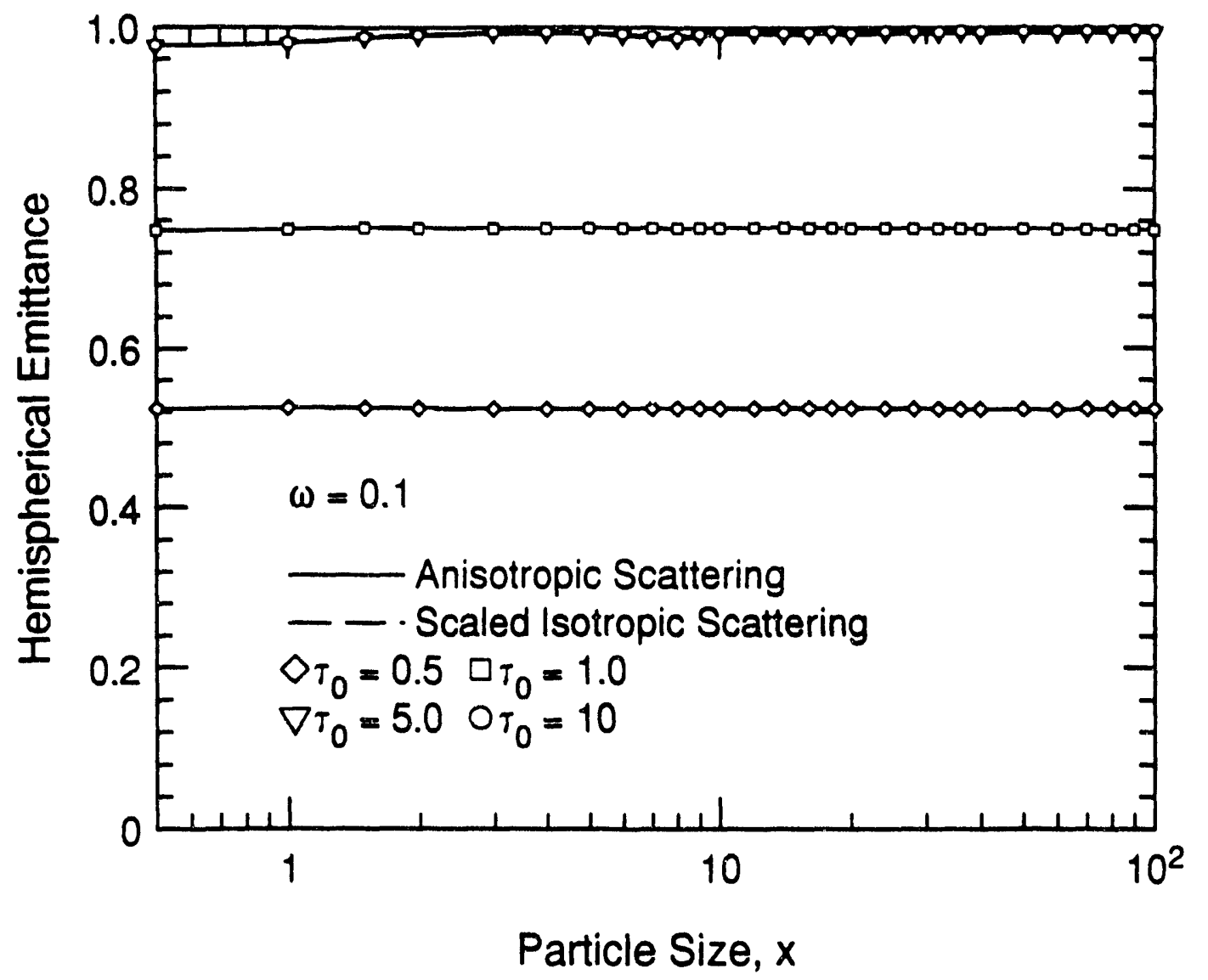

Figure 4: The hemispherical emittance versus particle size for scattering albedo, $\omega=0.1$, and various optical depths, $\tau_{0}$

with $M=128$, show excellent agreement with the results using "scaled" isotropic scattering, with the largest disc-epancy occurring for $\omega=0.9$ and $\tau_{0}=5.0$ in the range $1<x<5$. As expected, when the influence of multiple scattering is low $\left(\tau_{0} \omega<1\right)$ the scaled isotropic results are very accurate, but it is also evident that even when there are significant levels of multiple scattering $(\tau=10, \omega=0.9)$ the scaled isotropic model predicts the hemispherical emittance very well.

Figure 7 shows the computed hemispherical reflectance for $\omega=0.9$. The scaled isotropic model does not predict reflectance as accurately as it predicts emittance, with errors on the order of $5-8 \%$ for $\tau_{0}=0.5$ and less than approximately $1 \%$ for $\tau_{0}=10$. This small error is acceptable in most engineering situations. 


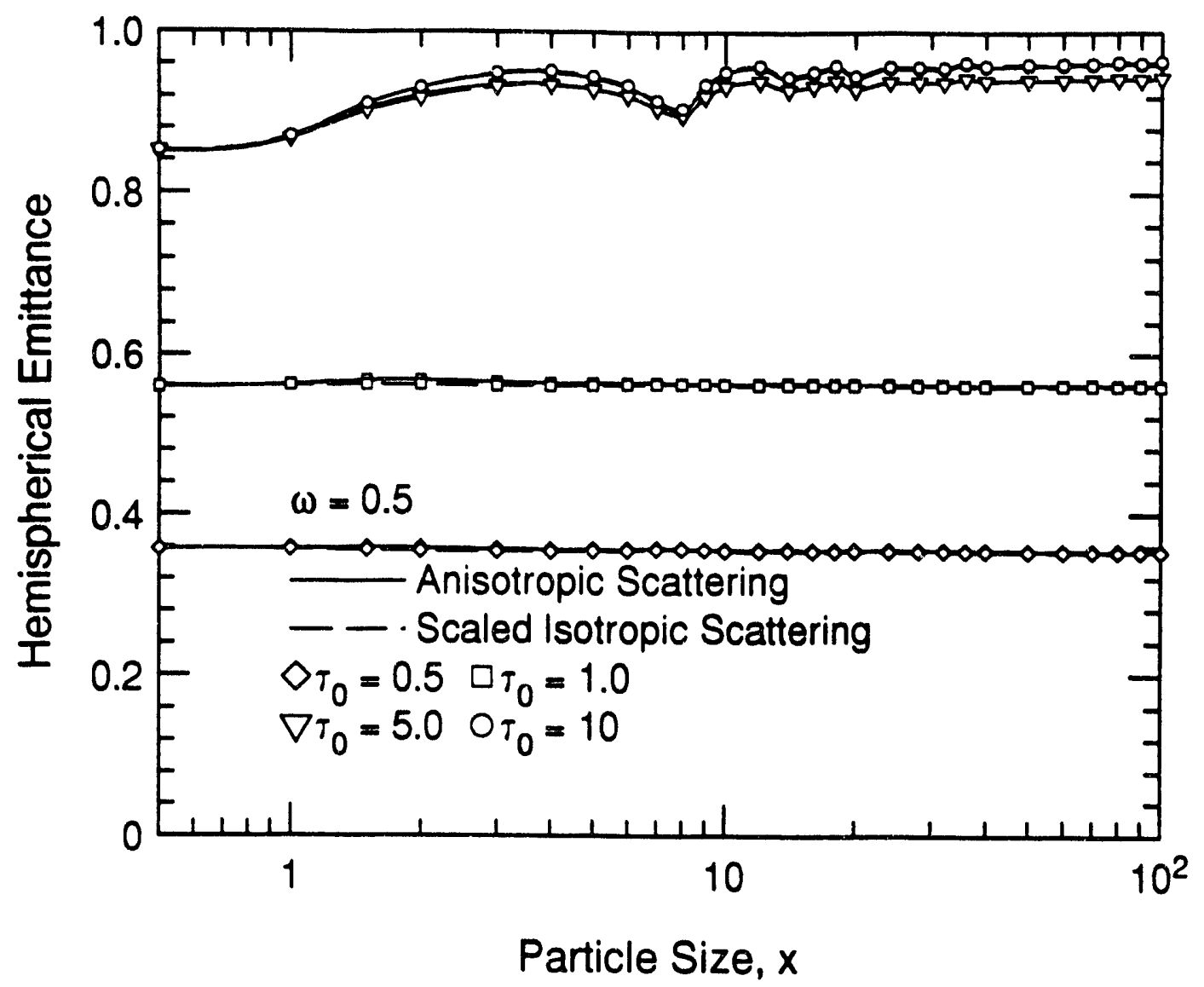

Figure 5: The hemispherical emittance versus particle size for scattering albedo. $\omega=0.5$, and various optical depths, $\tau_{0}$

The scaled isotropic model predicts hemispherical quantities very accurately, but does not predict directional emergent radiances as accurately. Figure 8 shows the directional reflectance for three different particle sizes. The scaled isotropic solution predicts the directional reflectance very accurately for $x=0.5$, which is to be expected since the scattering phase function is very symmetric and is closely represented by isotropic scattering. However, for larger $x$, the model phase function does not approximate the real phase function so well, and higher order models are required. The model phase function (isotropic plus a delta function) causes under-prediction of the reflectance at angles far the normal and over-prediction near the normal $(\theta=0)$. Similar trends (not presented) are seen for directional emittance and transmittance. 


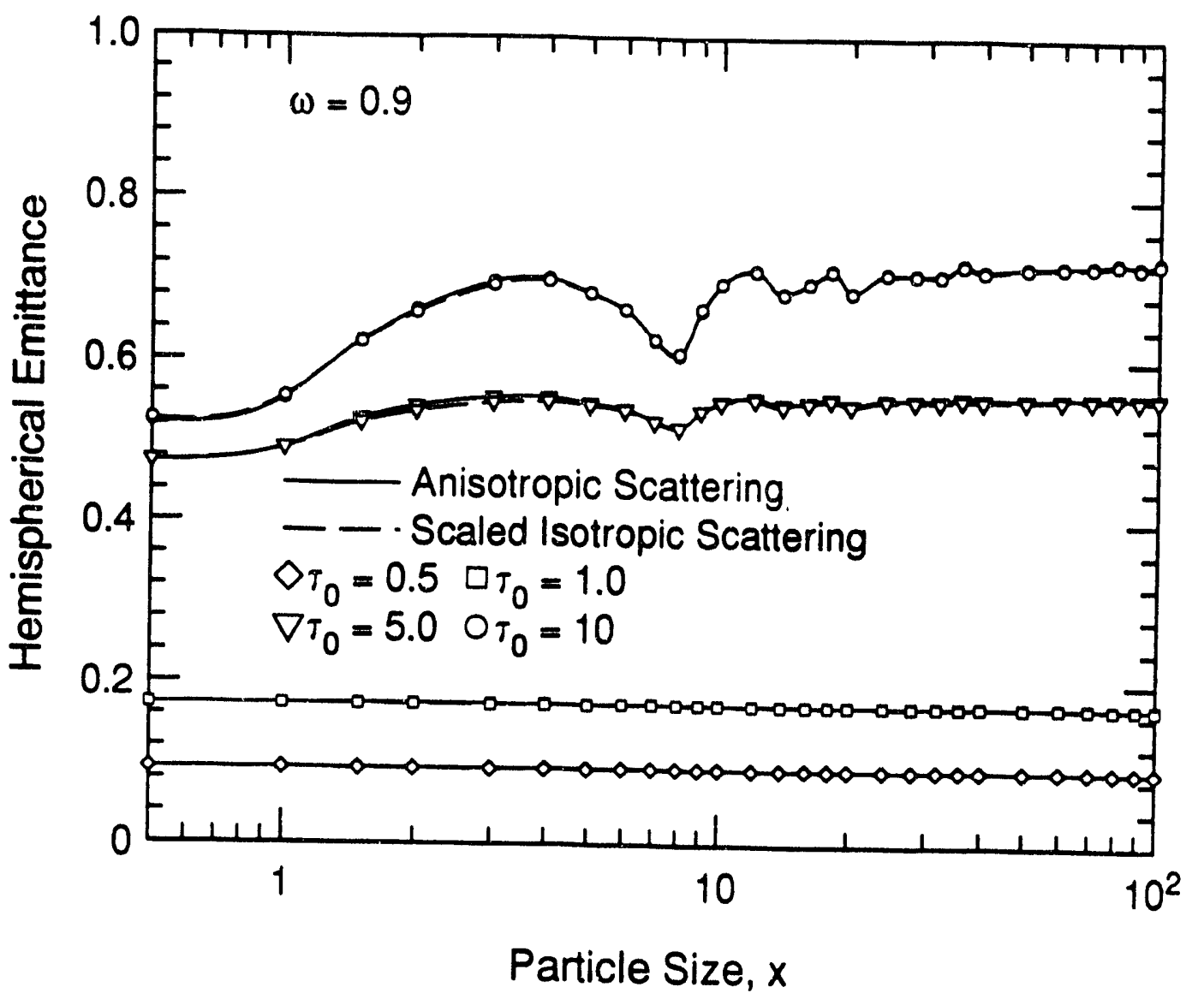

Figure 6: The hemispherical emittance versus particle size for scattering albedo, $\omega=0.9$, and various optical depths, $\tau_{0}$

During the past quarter, a new computer code was developed that allows calculation of the radiation heat transfer through a non-homogeneous anisotropically scattering medium. The code has been tested against exact solutions and published results and found to be accurate.

Several sample calculations were presented to illustrate the difficulties of computing radiation heat transfer through anisotropically scattering media. It was demonstrated that using a model scattering phase function consisting of an isotropic component plus a delta function yields accurate estimates of hemispherical quantities, such as heat transfer, but that directional quantities are poorly predicted if the particles are large with a highly asymmetric scattering phase function.

As results from tasks 1 and 2 become available. this computer code will be used to study the effects of fly ash on the radiation heat transfer in coal combustors. 


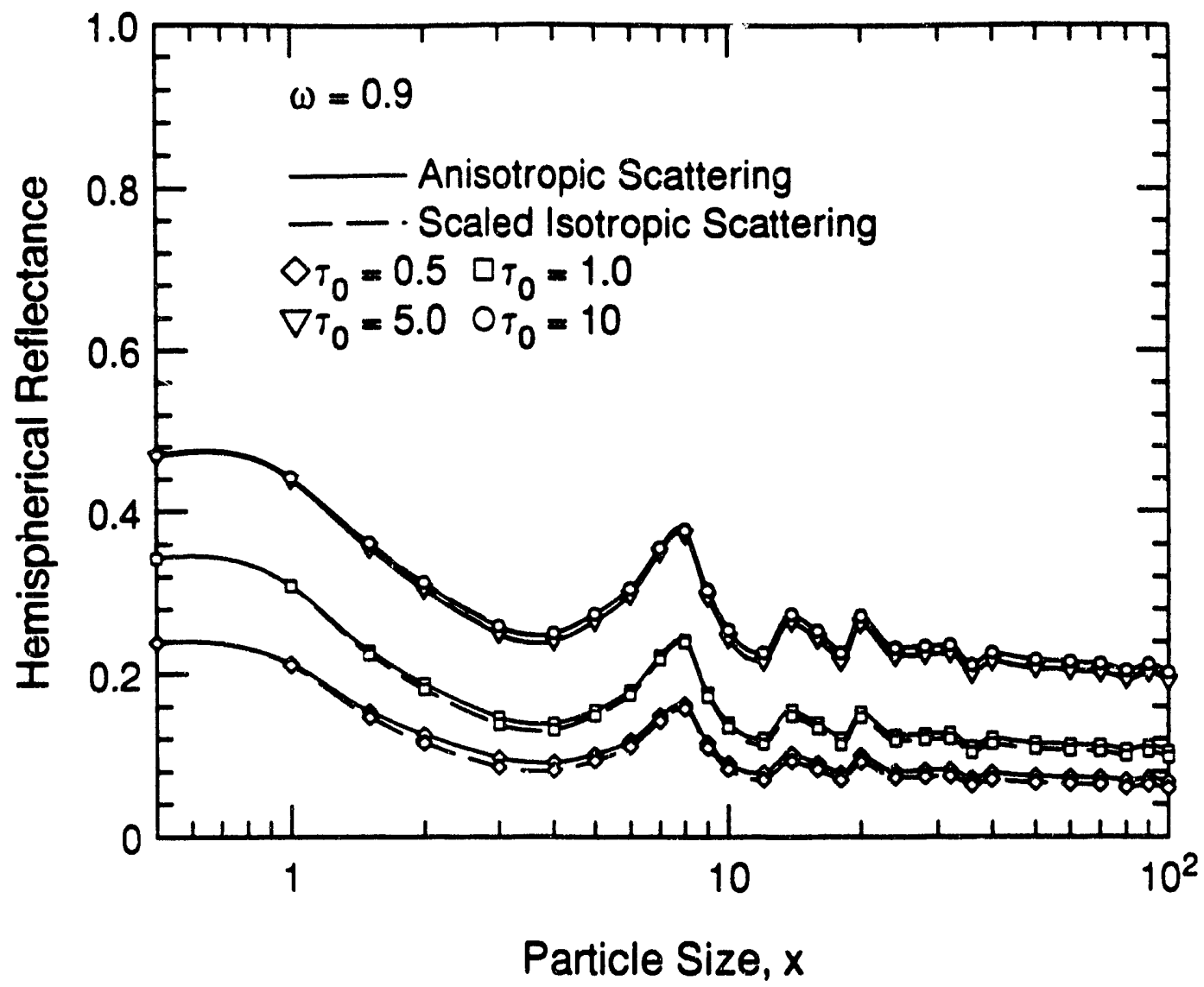

Figure 7: The hemispherical reflectance versus particle size for scattering albedo, $\omega=0.9$, and various optical depths, $\tau_{0}$ 


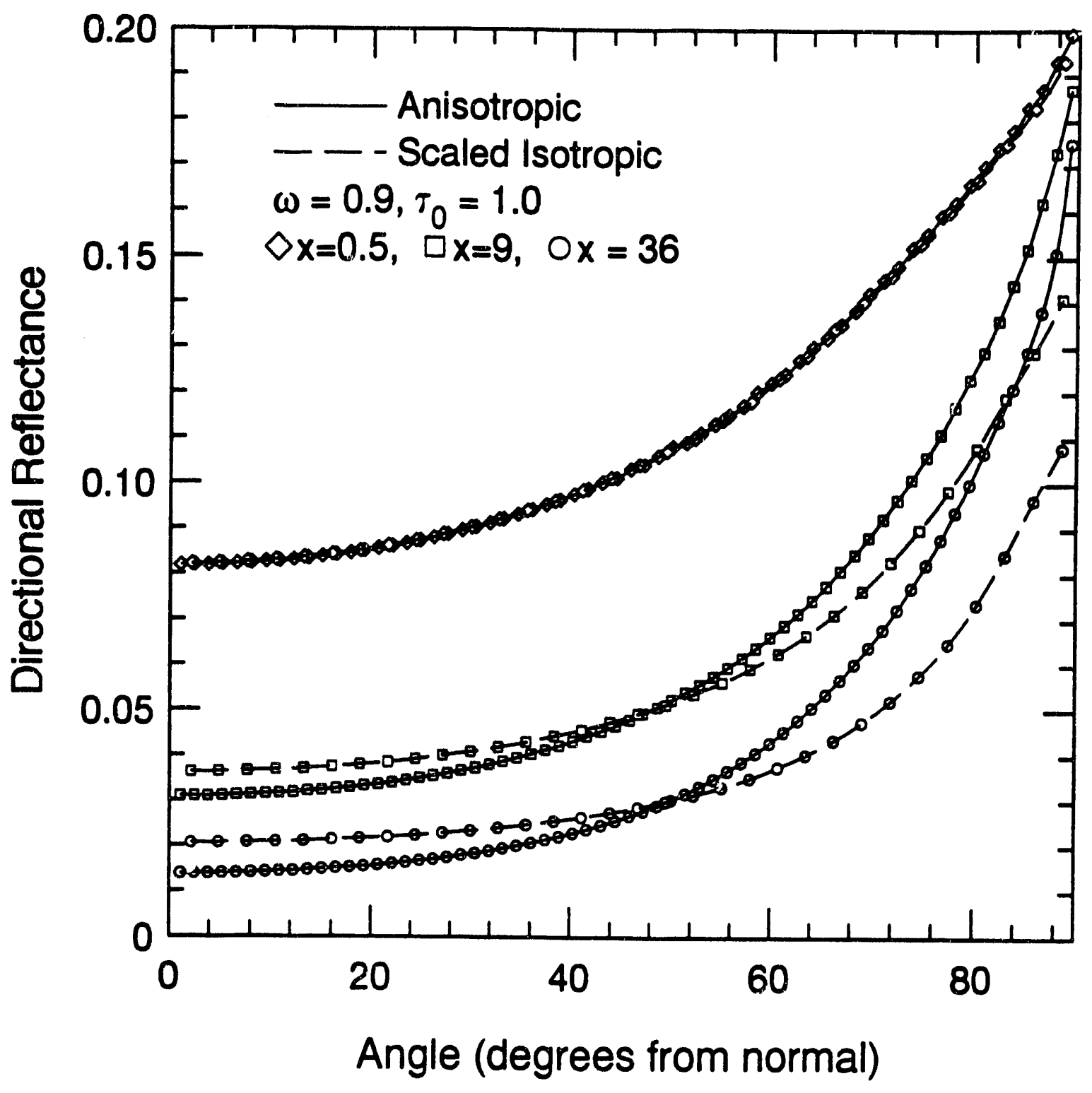

Figure 8: The directional reflectance for $\omega=0.9, \tau_{0}=1$, and $x=$ $0.5,9$, and 36 


\subsection{Task 4: Measurement of the Radiant Properties of Fly Ash Dispersions:}

Experiments leading to the final design of the test cell have been made throughout the previous quarter. Difficulties have been encountered in maintaining a steady transmitted optical signal through the ash dispersion in the cell. Improvements have been made by decreasing the stirrer speed to reduce turbulence, and also by immersing an extension of the cell in an ultrasonic bath to prevent re-agglommeration of ash. The use of ultrasonic agitation has also reduced the deposition of ash on the window surfaces. Further progress in overcoming these two problems is necessary, and work is continuing. 


\subsection{References}

1. J.L.Ebert and S.A.Self, "Radiation Heat Transfer in a Dispersion of Flyash", Engineering Foundation Conference on Mineral Matter and Ash Deposition from Coal, Santa Barbara, CA, Feb 22-26, 1988, pp.599-611.

2. C. F. Bohren and D. R. Huffman, Absorption and Scattering of Light by Small Particles, John Wiley \& Sons, 1983.

3. M. N. Özisik, Radiative Transfer and Interactions with Conduction and Convection, John Wiley \& Sons, 1973.

4. S. Chandrasekhar, Radiative Transfer, Dover 1960.

5. W. A. Fiveland, "Discrete-ordinates solutions of the radiative transport equation for rectangular enclosures", Journal of Heat Transfer 106, pp.699-706, 1984.

6. W. H. Press. B. P. Flannery, S. A. Teukolsky, and W. T. Vettering, Numerical Recipes - The Art of Scientific Computing, Cambridge University Press, 1986.

7. H. Lee and R. O. Buckius, "Scaling anisotropic scattering in radiation heat transfer for a planar medium", Journal of Heat Transfer, 104C, pp. 68-75, 1982.

8. R. P. Gupta. T. F. Wall, and J. S. Truelove, "Radiative scatter by fly ash in pulverized - coal - fired furnaces: application of the Monte Carlo method to anisotropic scatter", International Journal of Heat and Mass Transfer, 26, 1649-1660, 1983. 

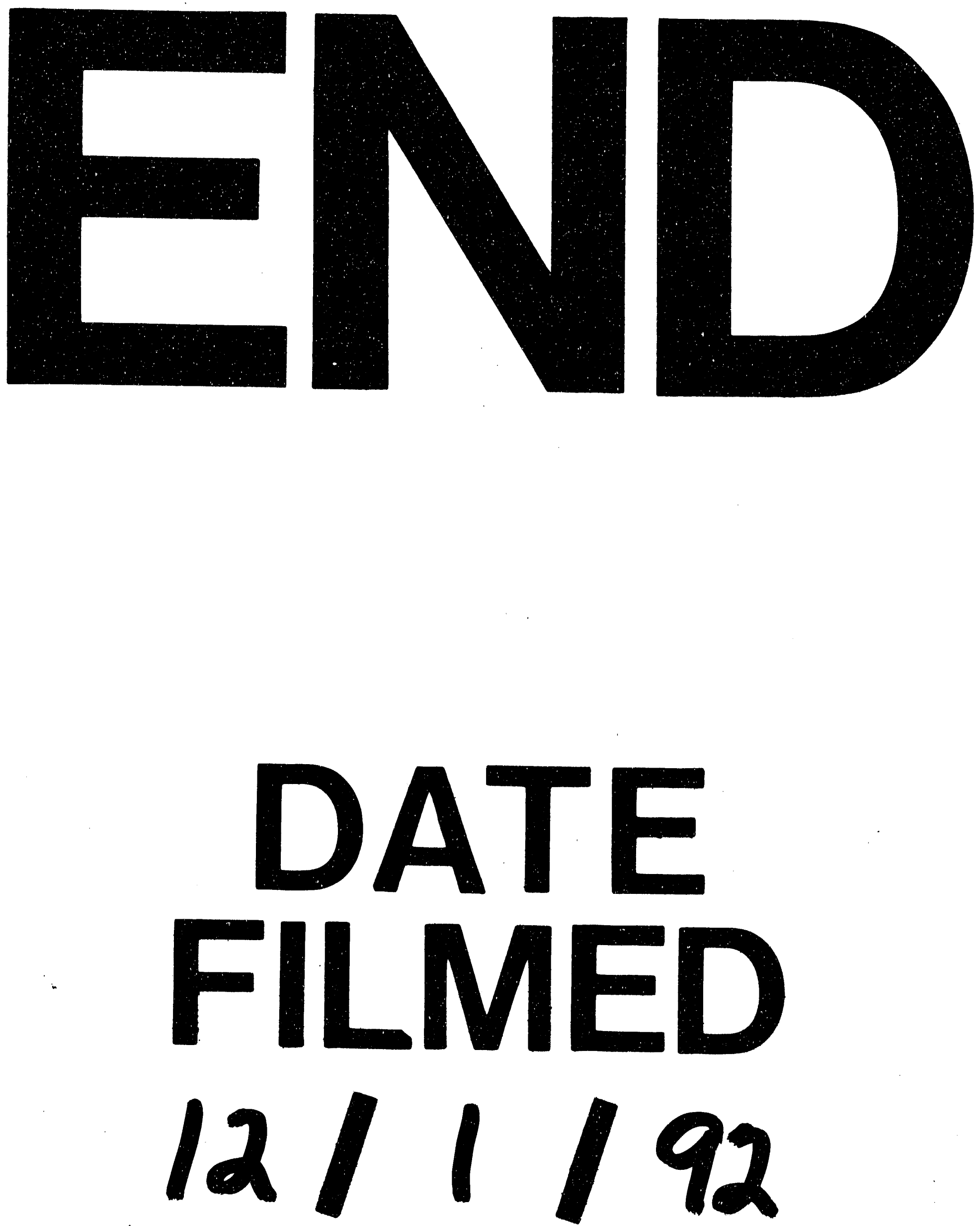
